\title{
A multiproxy database of western North American Holocene paleoclimate records
}

\author{
Cody C. Routson ${ }^{1}$, Darrell S. Kaufman ${ }^{1}$, Nicholas P. McKay ${ }^{1}$, Michael P. Erb ${ }^{1}$, Stéphanie H. Arcusa ${ }^{1}$, \\ Kendrick J. Brown ${ }^{2,3}$, Matthew E. Kirby ${ }^{4}$, Jeremiah P. Marsicek ${ }^{5}$, R. Scott Anderson ${ }^{1}$, \\ Gonzalo Jiménez-Moreno $^{6}$, Jessica R. Rodysill ${ }^{7}$, Matthew S. Lachniet ${ }^{8}$, Sherilyn C. Fritz ${ }^{9}$, \\ Joseph R. Bennett ${ }^{10}$, Michelle F. Goman ${ }^{11}$, Sarah E. Metcalfe ${ }^{12}$, Jennifer M. Galloway ${ }^{13}$, \\ Gerrit Schoups ${ }^{14}$, David B. Wahl ${ }^{15}$, Jesse L. Morris ${ }^{16}$, Francisca Staines-Urías ${ }^{17}$, Andria Dawson ${ }^{18}$, \\ Bryan N. Shuman ${ }^{19}$, Daniel G. Gavin ${ }^{20}$, Jeffrey S. Munroe ${ }^{21}$, and Brian F. Cumming ${ }^{22}$ \\ ${ }^{1}$ School of Earth and Sustainability, Northern Arizona University, P.O. Box 4099 Flagstaff, AZ 86011, USA \\ ${ }^{2}$ Canadian Forest Service, Natural Resources Canada, Victoria, BC V8Z 1M5, Canada \\ ${ }^{3}$ Department of Earth, Environmental and Geographic Sciences, University of British Columbia, \\ Okanagan, BC V1V 1V7, Canada \\ ${ }^{4}$ Department of Geological Sciences, California State University, Fullerton, 800 N. State College Blvd., \\ Fullerton, CA 98324, USA \\ ${ }^{5}$ Department of Geoscience, University of Wisconsin-Madison, 1215 W. Dayton St. Madison, WI 53706, USA \\ ${ }^{6}$ Departamento de Estratigrafía y Paleontología, Universidad de Granada, \\ Avda. Fuentenueva S/N, Granada 18002, Spain \\ ${ }^{7}$ Florence Bascom Geoscience Center, United States Geological Survey, \\ 12201 Sunrise Valley Dr. MS926A, Reston, VA 20192, USA \\ ${ }^{8}$ Department of Geoscience, University of Nevada, Las Vegas, \\ 4505 S. Maryland Parkway, Las Vegas, NV 89154, USA \\ ${ }^{9}$ Department of Earth and Atmospheric Sciences, University of Nebraska-Lincoln, Lincoln, NE 68540, USA \\ ${ }^{10}$ Department of Biology, Carleton University, 1125 Col By Drive, Ottawa, ON K1S 5B6, Canada \\ ${ }^{11}$ Department of Geography, Environment, and Planning, Sonoma State University, \\ 1801 E. Cotati Ave, Rohnert Park, CA 94928, USA \\ ${ }^{12}$ School of Geography, University of Nottingham, University Park, \\ Nottingham, Nottinghamshire, NG7 2RD, UK \\ ${ }^{13}$ Geological Survey of Canada (Commission géologique du Canada), 3303 33rd St. NW, \\ Calgary, AB T2L 2A7, Canada \\ ${ }^{14}$ Water Resources Management, Delft University of Technology, P.O. Box 5048, \\ Delft, 2600 GA, the Netherlands \\ ${ }^{15}$ Geology, Minerals, Energy, and Geophysics Science Center, United States Geological Survey, 345 \\ Middlefield Rd., Menlo Park, CA 94025, USA \\ ${ }^{16}$ Department of Geography, University of Utah, 260 Central Campus Dr \#4625, \\ Salt Lake City, UT 84112, USA \\ ${ }^{17}$ Department of Marine Geology, Geological Survey of Denmark and Greenland (GEUS), \\ Oester Voldgade 10, Copenhagen K, 1350, Denmark \\ ${ }^{18}$ Department of General Education, Mount Royal University, \\ 4825 Mt Royal Gate SW, Calgary, AB T3E6K6, Canada \\ ${ }^{19}$ Department of Geology and Geophysics, University of Wyoming, 1000 E. University Ave., \\ Laramie, WY 82071, USA \\ ${ }^{20}$ Department of Geography, University of Oregon, 1251 University of Oregon, Eugene, OR 97403, USA \\ ${ }^{21}$ Geology Department, Middlebury College, 276 Bicentennial Way, Middlebury, VT 05753, USA \\ ${ }^{22}$ Department of Biology, Queen's University, 116 Barrie St., Kingston, ON K7L3J9, Canada
}


Correspondence: Cody C. Routson (cody.routson@nau.edu)

\author{
Received: 29 July 2020 - Discussion started: 30 September 2020 \\ Revised: 31 January 2021 - Accepted: 10 February 2021 - Published: 19 April 2021
}

\begin{abstract}
Holocene climate reconstructions are useful for understanding the diverse features and spatial heterogeneity of past and future climate change. Here we present a database of western North American Holocene paleoclimate records. The database gathers paleoclimate time series from 184 terrestrial and marine sites, including 381 individual proxy records. The records span at least 4000 of the last 12000 years (median duration of 10725 years) and have been screened for resolution, chronologic control, and climate sensitivity. Records were included that reflect temperature, hydroclimate, or circulation features. The database is shared in the machine readable Linked Paleo Data (LiPD) format and includes geochronologic data for generating sitelevel time-uncertain ensembles. This publicly accessible and curated collection of proxy paleoclimate records will have wide research applications, including, for example, investigations of the primary features of oceanatmospheric circulation along the eastern margin of the North Pacific and the latitudinal response of climate to orbital changes. The database is available for download at https://doi.org/10.6084/m9.figshare.12863843.v1 (Routson and McKay, 2020).
\end{abstract}

\section{Introduction}

Reconstructing past climate is challenging because it is spatially and temporally complex and because all paleoclimate records are influenced by factors other than climate. Although rarely done, taking advantage of the full breadth of paleoclimatic evidence provides the best possibility of discerning signal from noise. Of all the geologic epochs, the paleoclimate of the Holocene (11.7 kiloannum (ka) to present) has been investigated most extensively. Studying the Holocene is useful, in part, because it serves as a baseline from which to assess natural versus human-forced climate changes. A keyword search on "Holocene" and "climate" returns approximately 21000 studies globally on the Web of Science. The volume of this previous work, as well as the evolving scientific understanding that it represents, generates organizational challenges related to data validation, extraction, and application.

Here we present a new database of Holocene paleoclimate records from western North America and the adjacent eastern Pacific Ocean. The spatial domain (Fig. 1) extends from tropical Mexico to Arctic Alaska. This region was chosen because (1) it encompasses the large latitudinal range necessary to study effects of orbital changes, the primary climate forcing during the Holocene; (2) it is affected by the major modes of modern Pacific climate variability including the Pacific Decadal Oscillation (Mantua et al., 1997), El Niño-Southern Oscillation (ENSO) (Redmond and Koch, 1991), and the Northern Annular Mode (McAfee and Russell, 2008), among others; (3) it represents a range of climatologies, especially hydroclimate as influenced by the Pacific westerlies and North American monsoon (Adams and Comrie, 1997); (4) it features multiple sources of proxy climate information, including marine sediment, caves, glaciers, and lakes, which are sensitive to changes in wintertime mois- ture, a key variable for tracking the primary variability of North Pacific ocean-atmospheric circulation; and (5) it is a region of concern for future climate change, considering the large population growth and climate hazards related to, for example, water scarcity in the southern tier (Garfin, 2013) and changing wildfire hazards throughout (e.g., Marlon et al., 2012; Power et al., 2008).

This database is composed of records from individual sitelevel studies and records that were compiled by previous summaries. Many (42\%) of the records in this database are also included in version 1 of the global Temperature $12 \mathrm{k}$ database (Kaufman et al., 2020a). This database adds another 39 temperature-sensitive records, plus 179 records that reflect hydroclimate and circulation changes. The added data were published in various formats and often with little metadata to inform the reuse of the data. Together, this geographically distributed collection of proxy climate records integrates marine and terrestrial realms and forms a network from which to assess the spatial variability of regional climatic change and ocean-atmospheric circulation and to compare with climate model simulations of past climate states.

\section{Data and methods}

\subsection{Data collection}

Paleoclimate records located in western North America and the adjacent Pacific Ocean (Fig. 1) were considered for inclusion in the database. They were obtained from public archives in PANGEA and NOAA's World Data Service (WDS) for Paleoclimatology using the keyword search "Holocene" and record duration searches on NOAA's paleoclimate search engine. The remainder were obtained through either the supplements of publications or directly from individual data generators and are now being made available in 

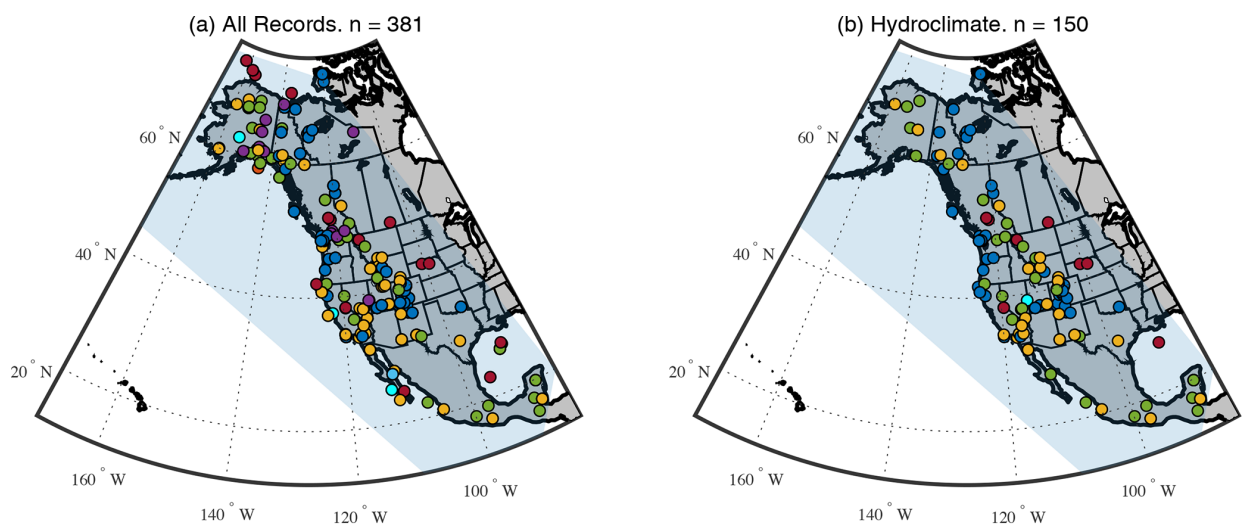

(c) Temperature. $\mathrm{n}=200$

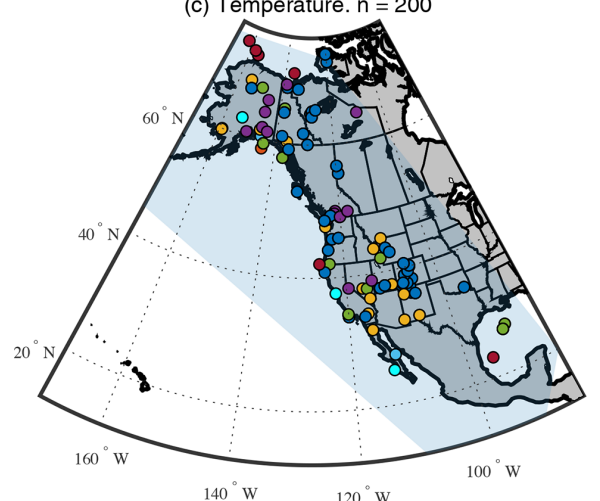

(d) Other Records. $n=31$

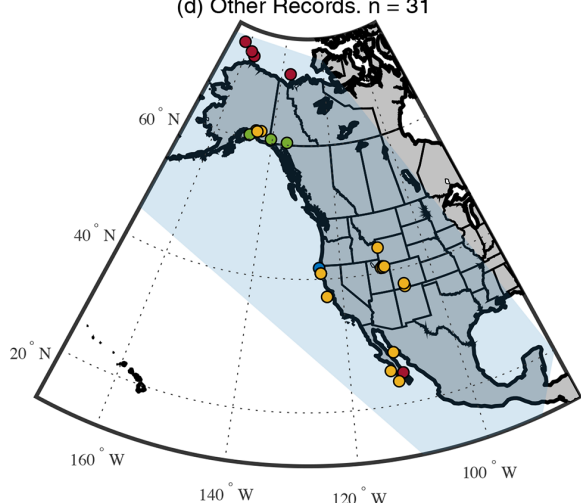

(e) Temporal Availability

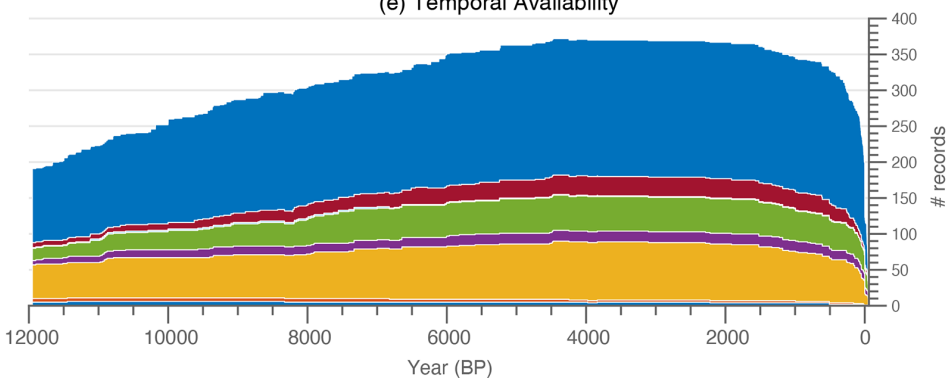

$\circ \mathrm{Mg} / \mathrm{Ca}$

- alkenone

○ biophysical

- chironomid

$\circ$ isotope

○ other biomarker

- other microfossil

- pollen

Figure 1. Spatiotemporal distribution of the western North American Holocene paleoclimate database. (a) The database includes 381 proxy records from a variety of archive and proxy types. Records include those in calibrated climate units (e.g., $\left.{ }^{\circ} \mathrm{C}\right)$ and records in their native proxy units (e.g., $\delta^{18} \mathrm{O}$ ). (b) Distribution of records sensitive to hydroclimate including precipitation, flood frequency, and $P-E(n=150)$. (c) Spatial distribution of the subset of records sensitive to temperature $(n=200)$ and (d) the spatial distribution of other records including upwelling, sea ice, glacier extent, dust, circulation, and climate modes $(n=31)$. (e) Temporal availability of the records in the database by proxy type (proxy general in Supplement Table S1) over the last $12 \mathrm{ka}$.

digital form as part of this data product. This database builds on several previously published paleoclimate data compilations overlapping the spatial domain encompassed by this study. These include the global Holocene temperature reconstruction of Marcott et al. (2013) $(n=4$ records in western North America), Arctic Holocene Transitions database (Sundqvist et al., 2014) $(n=30$ records in western North America), a collection compiled to characterize Holocene North American monsoon variability (Metcalfe et al., 2015) ( $n=8$ records in common with this database), the Northern Hemisphere dataset used to reconstruct Holocene temperature gradients and mid-latitude hydroclimates (Routson et al., 2019a) ( $n=55$ records in common with this database), a network of Holocene pollen reconstructions (Marsicek et al., 2018) ( $n=71$ records in common with this study), two collections of records focused on the last 2 millennia (Rodysill et al., 2018; Shuman et al., 2018) $(n=18$ and $n=16$ records in common with this study respectively), and the global Temperature 12k database (Kaufman et al., 2020a) ( $n=161$ records in common with this database). Two dust deposition records were included from the global dust compilation (Albani et al., 2015). This database also complements the recently published PAGES (Past Global Changes) global multiproxy database for temperature reconstructions of the 
Common Era (PAGES 2k Consortium, 2017) and the PAGES global database for water isotopes over the Common Era (Konecky et al., 2020), which are both structured in the same format as this database. A few of the records were not available from the original data generators, and therefore the time series data were digitized from the source publication (as noted in the metadata) using the MATLAB program digitize2.m (Anil, 2020). Digitized records were mainly included to fill geographic gaps in the network of proxy sites.

Other Holocene paleoclimate records were considered but ultimately excluded because they did not satisfy the selection criteria. The majority of excluded records either (1) lacked a clear relation between proxy and climate, (2) were of insufficient duration, (3) possessed large gaps between chronologic control points, or (4) did not meet the sampling resolution criteria. In some instances selection criteria were eased to fill geographic gaps or for reasons justified by the authors in the QC (quality control) comments metadata. Removing records from the database for subjective reasons, such as removing records with outliers, was avoided.

\subsection{Relation between proxy and climate}

Only records with a demonstrated relation to a climate variable were included, as interpreted by the original authors of the site-level studies, but some records are not calibrated to a climate variable. Calibrated records, for example, are presented in temperature units $\left({ }^{\circ} \mathrm{C}\right)$ and precipitation units $(\mathrm{mm})$. Other records are reported in their native proxy variables (e.g., $\delta^{18}, \%$, or sediment mass accumulation, $\mathrm{g} / \mathrm{cm}^{2} / \mathrm{yr}$ ). Some calibrated records rely on statistical procedures to determine the relationship between proxy and instrumental data and to infer paleoclimate change, assuming that the processes that control the proxy signal remain constant down core (Tingley et al., 2012; Von Storch et al., 2004). Other calibrations rely on transfer functions based on the correlation of contemporary environmental gradients (e.g., Juggins and Birks, 2012) or the modern analogue technique, which uses the similarity between modern and fossil assemblages (e.g., Guiot and de Vernal, 2007). The original species assemblage data (primarily pollen) for these records are not included in this data product. However, a link to the Neotoma Paleoecology Database dataset ID is provided where available. The Neotoma Paleoecology Database is a community-curated database that is a primary repository for assemblage and other paleoecology data (Williams et al., 2018).

The database also includes proxy records that have not been calibrated to a specific climate variable but that display a clear relation between the proxy and climate. These "relative" climate indicators are useful because they (1) attest to the timing and relative magnitude of change, which is sufficient for many statistical reconstruction methods, especially those that do not assume linearity between proxy and climate variables; (2) can be used in proxy system modeling and in some cases (e.g., $\left.\delta^{18} \mathrm{O}\right)$ can be compared directly to the output of climate models; and (3) provide more complete spatial coverage.

\subsection{Record duration and resolution}

The database aims to document paleoclimate variability that ranges on the timescale of multi-millennial trends to centennial excursions. However, not all records encompass the entire Holocene epoch. To be included, records must span a duration of ca. 4000 years anytime between 0 and $12 \mathrm{ka}$. To focus on records that can resolve sub-millennial patterns, the database includes those with a sample resolution finer than 400 years (i.e., the median spacing between consecutive samples in the time series is less than 400 years over the past 12000 years or over the full record length, if shorter).

\subsection{Chronologic control}

Age control is a fundamental variable underlying proxy records. The database includes the chronologic data necessary for reproducing original age-depth models for records from sediment and speleothem archive types. Chronologic data include depth, uncalibrated radiometric or other dates, analytical errors, and associated corrections where applicable. Other metadata, including material type analyzed and sample identifiers, were included when available. Time series with a maximum of 3000 years between dates within the $0-12 \mathrm{ka}$ interval or with five or more relatively evenly distributed Holocene dates were included in the database. Overall, the age control screening retained a high proportion of available records while recognizing that such coarse age control often precludes the ability to address questions that require fine temporal-scale accuracy (Blaauw et al., 2018).

\subsection{Metadata}

The database includes a large variety of metadata (Supplement Table S1) to facilitate analyses and reuse. The metadata included in this database are largely consistent with those developed and used in the Temperature 12k database (Kaufman et al., 2020a), with some refinement for hydroclimate-related records. Predominant metadata are subdivided into the following categories:

1. Geographic information includes "site name", "latitude", "longitude", and "elevation". Geodetic data are relative to the WGS84 (World Geodetic System 1984) ellipsoid and in units of decimal degrees. "Country ocean" is generated based on the NASA GCMD (Global Change Master Directory) convention.

2. Bibliographic information includes the DOI (digital object identifier) when available. The original study is typically referenced in "publication 1". "Publication 2" generally corresponds to subsequent publications contributing to record development or reuse. 
3. The original data source ("original data citation") is the persistent identifier (URL, Uniform Resource Locator, or DOI) that connects to the publicly accessible repository (e.g., PANGAEA and NOAA WDS paleoclimatology when available). Fields with the entry "wNAm" correspond to records transferred to a public repository for the first time by this study. "Neotoma ID" includes the Neotoma dataset ID when available for the original assemblage data.

4. Metadata describing the proxy record include "archive type", "proxy general", "proxy type", "proxy detail", "calibration method", and "paleo data notes". Archive type corresponds to the physical archive (e.g., lake sediment, marine sediment, peat, and speleothem). Proxy general simplifies plotting figures by grouping similar proxies from proxy type. For example, proxy general for "other biomarkers" includes proxy type TEX86 (tetraether index of 86 carbon atoms) and GDGT (glycerol dialkyl glycerol tetraether) but not alkenones, which are treated separately. Proxy general for "biophysical" includes biogenic silica, tree-ring width, total organic content, chlorophyll, and macrofossils. Proxy general for "other microfossil" includes coccolith, diatom, dinocyst, and foraminifera. Pollen and chironomid records are treated separately. Proxy detail corresponds to specific species or material types. "Calibration method" is the statistical method used for proxy calibration. Paleo data notes include information from the original study to help users understand the proxy record.

5. For climate interpretation, primary "climate variables" include " $T$ " (temperature), " $P$ " (precipitation), and " $P-E$ " (precipitation minus evaporation). Other climate indicators include "MODE" (climate modes such as ENSO), "upwelling" (coastal upwelling), "DUST" (dust deposition), "ICE" (sea ice extent), and "ELA" (glacier equilibrium line altitude). The "interpretation direction" is the sign relation ("positive" or "negative") between the proxy value and the climate variable. Proxy records originally reported as $E-P$ were cataloged as the climate variable of $P-E$, and the field interpretation direction was inverted from the original interpretation. "Variable name" corresponds to the specific variable type (e.g., "temperature" or " ${ }^{18} \mathrm{O}$ "; oxygen18 isotopes). "Units" correspond to the measurement unit specified in the variable name (e.g., " $\operatorname{deg} C$ " or "permil"). "Climate variable detail" refines the climate variable field. Temperature records follow the structure of the variable sensed (e.g., "air") at a specific level (e.g., "surface"). Examples include "air@ surface", "air@condensation", and “sea@surface”. Hydroclimate and some other record types do not always conform as well to this format. Climate variable detail for these records specifies the variable sensed (e.g., "lake level", "runoff", "river flow", and "amount"), at a specific level (e.g., surface). Examples include "lakeLevel@ surface" and "runoff@surface". If the variable sensed is the same as the climate variable (e.g., "precipitation"), the field is left blank. In these cases only the level is specified (e.g., "@surface”). In cases where the level was ambiguous, not specified, or not applicable (e.g., "soil moisture", "lake salinity", or "El Niño"), only the variable sensed was specified.

6. Seasonality information has been separated into two fields of "seasonality" and "seasonality general". Seasonality includes the most specific seasonal information available including specific months in number format (July $=$ "7") or reconstructed seasons (e.g., "warmest month", "summer", "growing season", "winter", and "annual"). "Season general" distills season details into queryable seasons ("annual", "summer only", "summer+", "winter only", and "winter+"). Categories summer+ and winter+ indicate that another season (or annual) has also been reconstructed from the same site.

7. Metadata describing the underlying time series data include the youngest and oldest sample ages ("min year" and "max year"), the median sample resolution ("resolution") over the past 12000 years, and the frequency of age control points ("ages per kyr"), which includes radiocarbon and U-series (uranium) ages.

8. Quality control metadata include ("QC certification") and ("QC comments"). QC certification includes the initials of the co-author of this data descriptor who was responsible for reviewing the screening criteria for records included in the data product. QC comments were written by the person who completed QC to improve reusability of the data.

9. Data access and visualization includes a website link for viewing and downloading the data in .csv (commaseparated value) or LiPD format ("link to LiPDverse").

\subsection{Database structure and format: Linked Paleo Data (LiPD)}

The site-level data and metadata are formatted in the LiPD structure. The LiPD framework comprises JSONformatted files that are machine-readable with MATLAB, Python, and $\mathrm{R}$ packages that enable rapid querying and data extraction (McKay and Emile-Geay, 2016). LiPD encodes the database into a structured hierarchy that allows for explicit descriptions at any level and aspect of the database. Code packages for evaluating the database can be accessed on GitHub (https://github.com/nickmckay/ LiPD-utilities, last access: 29 March 2021). 


\subsection{Data visualization}

A one-page dashboard for each record is included as a Supplement to this article. The dashboards include the primary information associated with each record including the location, the time series plot, bibliographic reference, and proxy data information (Supplemental dashboards). Each record is also linked to a web page (link to LiPDverse) where the data can be visualized and downloaded in LiPD or text versions. A globally distributed collection of paleoclimate LiPD files is housed at https://lipdverse.org/ (last access: 29 March 2021). This western North American Holocene paleoclimate database is a subset of the records that can be found by choosing wNAm in the LiPDverse browser. The full collection can also be accessed at http://lipdverse.org/ wNAm/1_0_0/ (last access: 29 March 2021).

\section{Summary of database contents}

\subsection{Proxy records and climate variables}

The western North American Holocene paleoclimate database includes proxy climate records from 184 different sites. Many "sites" (locations) are represented by more than one proxy "record" (time series). Multiple records from one site often represent different climate variables or reconstruction methods. Pollen assemblages, for example, are often translated into both temperature and moisture variables, sometimes for different seasons. The list of sites is shown by row in Table 1, whereas Supplement Table S1 contains a row for each record. In total, this database comprises 184 sites and 381 records.

The records are derived from nine archive types and are based on eight proxy categories (Supplement Table S1). The database includes 259 records from lake sediments, 58 records from marine sediment, and 64 other terrestrial.

The western North America database includes 84 records that are being transferred to a publicly accessible data repository for the first time with this data product. These include 61 "new" records as follows. Pollen ratio time series reflecting changes in the position of forest boundaries and long-term temperature change were calculated for 23 records. These ratios were computed by the original data generators following methods and rationale described in Jiménez-Moreno et al. (2019) and Johnson et al. (2013). The database also includes 20 precipitation records, which were generated by Marsicek et al. (2018) but not released with that publication. Finally, we have included 18 hydroclimate records based on subsets of packrat midden sites from Harbert and Nixon (2018), following the same methods applied for temperature reconstructions in Kaufman et al. (2020b). Briefly, the Climate Reconstruction Analysis using Coexistence Likelihood Estimation (CRACLE) method was used to infer absolute precipitation given the modern relationship between WorldClim climate data and packrat midden fos- sil data. In the original paper (Harbert and Nixon, 2018), an overall MAT (mean annual temperature) anomaly that combines all sites is presented. This MAT is calculated by subtracting the WorldClim calibration data for each site and then averaging all inferred temperatures (across space) in discrete time intervals. Here we provide the absolute precipitation from CRACLE, without spatiotemporal averaging, and note that some of the inferred absolute precipitation appears more extreme than precipitation reconstructed from other proxies. For further details and code, please refer to Harbert and Nixon (2018). These midden records are noted in the QC comments column of Supplement Table S1.

The database contains 200 temperature-sensitive records; 150 hydroclimate sensitive records (e.g., precipitation, $P-E$, flood frequency, and streamflow); and 31 other records including upwelling, dust, climate mode, and sea ice extent. Marine records are primarily sea surface temperatures, but there are several marine records of other variables including sea ice extent, upwelling strength, and flood frequency. Many (228) of the proxy records are interpreted by the original authors to represent mean annual values of specific climate variables. Others represent individual seasons, primarily with some aspect of summer. Background information including the strengths, weaknesses, and underlying assumptions of the specific poxy types can be found in textbooks devoted to the topic (e.g., Bradley, 2015).

\subsection{Geographic coverage}

The geographic distribution of records within western North America is far from uniform (Fig. 1). The density of all sites is comparatively high in Alaska and the conterminous western United States. In contrast, Mexico is represented by few study sites, mainly because many studies failed to meet the inclusion criteria. Hydroclimate records have the most uniform coverage, albeit with a spatial gap in Mexico. The spatial distribution of temperature records has gaps in Canada, the midwestern United States, Texas, and continental Mexico.

\subsection{Record length and temporal resolution}

Median record duration is 10725 years, not counting the duration of records beyond 12000 years. Most of the records (94\%) extend back at least 6000 years, thereby including the frequently modeled 6 ka paleoclimate time slice. The median sample resolution of individual records in the database is 127 years (Fig. 2).

\subsection{Geochronology}

Original geochronologic data for each record are included in the database. The database includes 2353 individual age control points $\left({ }^{14} \mathrm{C},{ }^{210} \mathrm{~Pb}\right.$, tephras, etc.). Tree-ring age control points (two studies) were excluded from this number. 
Table 1. Proxy records included in the database, listed alphabetically. See Supplement Table S1 for expanded metadata and links to the proxy time series and chronology data.

\begin{tabular}{|c|c|c|c|c|c|c|}
\hline Site name & Lat & Long & Archive type & Proxy* & Original data citation (last access: 29 March 2021) & Reference \\
\hline $3 \mathrm{M}$ Pond & 49.98 & -121.22 & LakeSediment & Chironomid & http://www.ncdc.noaa.gov/paleo/study/27330 & Pellatt et al. (2000) \\
\hline $893 \mathrm{~A}$ & 34.29 & -120.04 & MarineSediment & $\delta^{18} \mathrm{O}$ & http://www.ncdc.noaa.gov/paleo/study/27330 & Kennett et al. (2007) \\
\hline Abalone & 33.96 & -119.98 & LakeSediment & Pollen & http://www.ncdc.noaa.gov/paleo/study/27330 & Cole and Liu (1994) \\
\hline Alfonso Basin & 24.65 & -110.60 & MarineSediment & Coccolith & wNAm & Staines-Urías et al. (2015) \\
\hline Andy & 64.65 & -128.08 & LakeSediment & Pollen & http://www.ncdc.noaa.gov/paleo/study/15444 & Szeicz et al. (1995) \\
\hline Bald Lake & 40.87 & -110.49 & LakeSediment & $\mathrm{Eu} / \mathrm{Zr}$ & wNAm & Munroe et al. (2020) \\
\hline Banks Island (74MS12) & 72.37 & -119.83 & LakeSediment & Pollen & http://www.ncdc.noaa.gov/paleo/study/27330 & Gajewski et al. (2000) \\
\hline Banks Island (74MS15) & 73.53 & -120.22 & LakeSediment & Pollen & http://www.ncdc.noaa.gov/paleo/study/27330 & Gajewski et al. (2000) \\
\hline Battleground & 45.80 & -122.49 & LakeSediment & Pollen & http://www.ncdc.noaa.gov/paleo/study/27330 & Barnosky (1985b) \\
\hline Beaver Lake & 42.46 & -100.67 & LakeSediment & Diatom & http://www.ncdc.noaa.gov/paleo/study/23075 & Schmieder et al. (2011) \\
\hline Beef Pasture & 37.47 & -108.16 & LakeSediment & Pollen & http://www.ncdc.noaa.gov/paleo/study/27330 & Petersen (1985) \\
\hline Begbie Lake & 48.59 & -123.68 & LakeSediment & Pollen & wNAm & Brown et al. (2019) \\
\hline Bells Lake & 65.02 & -127.48 & LakeSediment & Pollen & https://doi.org/10.21233/N35G6P & Szeicz et al. (1995) \\
\hline Big Lake & 51.67 & -121.45 & LakeSediment & Diatom & http://www.ncdc.noaa.gov/paleo/study/23089 & Cumming et al. (2002) \\
\hline Bison Lake & 39.76 & -107.35 & LakeSediment & $\delta^{18} \mathrm{O}$ & http://www.ncdc.noaa.gov/paleo/study/10749 & L. Anderson (2011) \\
\hline Blue Lake & 37.24 & -106.63 & LakeSediment & XRF & http://www.ncdc.noaa.gov/paleo/study/27078 & Routson et al. (2019b) \\
\hline Boomerang Lake & 49.18 & -124.16 & LakeSediment & Pollen & wNAm & Brown et al. (2006) \\
\hline Boone & 55.58 & -119.43 & LakeSediment & Pollen & http://www.ncdc.noaa.gov/paleo/study/27330 & White and Mathewes (1986) \\
\hline Candelabra Lake & 61.68 & -130.65 & LakeSediment & Pollen & http://www.ncdc.noaa.gov/paleo/study/15444 & Cwynar and Spear (2007) \\
\hline Carleton Lake & 64.26 & -110.10 & LakeSediment & Chironomid & http://www.ncdc.noaa.gov/paleo/study/16296 & Upiter et al. (2014) \\
\hline Carp & 45.92 & -120.88 & LakeSediment & Pollen & http://www.ncdc.noaa.gov/paleo/study/27330 & Barnosky (1985a) \\
\hline Cascade Fen & 37.65 & -107.81 & LakeSediment & Pollen & http://www.ncdc.noaa.gov/paleo/study/27330 & Maher (1963) \\
\hline Castor Lake & 48.54 & -119.56 & LakeSediment & Reflectance & http://www.ncdc.noaa.gov/paleo/study/10310 & Nelson et al. (2011) \\
\hline Castor Lake & 48.54 & -119.56 & LakeSediment & $\delta^{18} \mathrm{O}$ & http://www.ncdc.noaa.gov/paleo/study/10310 & Nelson et al. (2011) \\
\hline Chichancanab Lake & 19.83 & -88.75 & LakeSediment & $\mathrm{CaCO}_{3}$ & http://www.ncdc.noaa.gov/paleo/study/5483 & Hodell et al. (1995) \\
\hline Chichancanab Lake & 19.83 & -88.75 & LakeSediment & $\mathrm{S}$ & http://www.ncdc.noaa.gov/paleo/study/5483 & Hodell et al. (1995) \\
\hline Chichancanab Lake & 19.83 & -88.75 & LakeSediment & $\delta^{18} \mathrm{O}$ & http://www.ncdc.noaa.gov/paleo/study/5483 & Hodell et al. (1995) \\
\hline Chihuahuenos Bog & 36.05 & -106.51 & Peat & Pollen & wNAm & R. S. Anderson et al. (2008a) \\
\hline Chitina Loess & 61.54 & -144.38 & Loess & Particle size & http://www.ncdc.noaa.gov/paleo/study/20529 & Muhs et al. (2013) \\
\hline Cleland Lake & 50.83 & -116.39 & LakeSediment & $\delta^{18} \mathrm{O}$ & http://www.ncdc.noaa.gov/paleo/study/21250 & Steinman et al. (2016) \\
\hline Cleland Lake & 50.83 & -116.39 & LakeSediment & $\delta^{18} \mathrm{C}$ & http://www.ncdc.noaa.gov/paleo/study/21250 & Steinman et al. (2016) \\
\hline Copley & 38.87 & -107.08 & LakeSediment & Pollen & http://www.ncdc.noaa.gov/paleo/study/27330 & Fall (1997) \\
\hline Corser Bog & 60.53 & -145.45 & Peat & GDGT & http://www.ncdc.noaa.gov/paleo/study/15444 & Nichols et al. (2014) \\
\hline Corser Bog & 60.53 & -145.45 & Peat & $\delta \mathrm{D}$ & http://www.ncdc.noaa.gov/paleo/study/15444 & Nichols et al. (2014) \\
\hline Cottonwood Pass Pond & 38.83 & -106.41 & LakeSediment & Pollen & http://www.ncdc.noaa.gov/paleo/study/27330 & Fall (1997) \\
\hline Crater Lake & 37.67 & -106.69 & LakeSediment & Particle size & wNAm & Arcusa et al. (2020) \\
\hline Crevice Lake & 45.00 & -110.58 & LakeSediment & $\delta^{18} \mathrm{O}$ & wNAm & Whitlock et al. (2012) \\
\hline Crevice Lake & 45.00 & -110.58 & LakeSediment & $\mathrm{CaCO}_{3}$ & wNAm & Whitlock et al. (2012) \\
\hline Cueva Diablo & 18.18 & -99.92 & Speleothem & $\delta^{18} \mathrm{O}$ & http://www.ncdc.noaa.gov/paleo/study/10670 & Bernal et al. (2011) \\
\hline Cumbres Bog & 37.02 & -106.45 & LakeSediment & Pollen & wNAm & Johnson et al. (2013) \\
\hline Dempster Hwy Peatland & 65.21 & -138.32 & Ice-other & $\delta^{18} \mathrm{O}$ & http://www.ncdc.noaa.gov/paleo/study/27330 & Porter et al. (2019) \\
\hline DJ6-93SF-6 & 37.63 & -122.37 & MarineSediment & $\mathrm{Mg} / \mathrm{Ca}$ & wNAm & McGann (2008) \\
\hline DSDP (Deep Sea Drilling Project) Site 480 & 27.90 & -111.65 & MarineSediment & Diatom & http://www.ncdc.noaa.gov/paleo/study/5855 & Barron et al. (2004) \\
\hline DSDP Site 480 & 27.90 & -111.65 & MarineSediment & $\mathrm{BSi}$ & http://www.ncdc.noaa.gov/paleo/study/5855 & Barron et al. (2004) \\
\hline Dune & 64.42 & -149.90 & LakeSediment & $\delta^{18} \mathrm{C}$ & http://www.ncdc.noaa.gov/paleo/study/13076 & Finney et al. (2012) \\
\hline Eldora Fen & 39.94 & -105.58 & LakeSediment & Pollen & http://www.ncdc.noaa.gov/paleo/study/27330 & No publication on record \\
\hline Eleanor Lake & 47.68 & -124.02 & LakeSediment & $\mathrm{BSi}$ & wNAm & Gavin et al. (2011) \\
\hline Emerald Lake & 39.15 & -106.41 & LakeSediment & Stratigraphy & http://www.ncdc.noaa.gov/paleo/study/23079 & Shuman et al. (2014) \\
\hline Emerald Lake & 39.15 & -106.41 & LakeSediment & Pollen & wNAm & Jiménez-Moreno et al. (2019) \\
\hline EN32_PC6 & 26.95 & -91.35 & MarineSediment & $\mathrm{Mg} / \mathrm{Ca}$ & http://www.ncdc.noaa.gov/paleo/study/27330 & Flower et al. (2004) \\
\hline EN32_PC6 & 26.95 & -91.35 & MarineSediment & $\delta^{18} \mathrm{O}$ & http://www.ncdc.noaa.gov/paleo/study/27330 & Flower et al. (2004) \\
\hline Enos Lake & 49.28 & -124.15 & LakeSediment & Pollen & wNAm & Brown et al. (2006) \\
\hline EW0408_66JC & 57.87 & -137.10 & MarineSediment & Alkenone & http://www.ncdc.noaa.gov/paleo/study/22400 & Praetorius et al. (2015) \\
\hline EW0408_66JC & 57.87 & -137.10 & MarineSediment & $\delta^{18} \mathrm{O}$ & http://www.ncdc.noaa.gov/paleo/study/22400 & Praetorius et al. (2015) \\
\hline EW0408_85JC & 59.56 & -144.15 & MarineSediment & Alkenone & http://www.ncdc.noaa.gov/paleo/study/21950 & Praetorius et al. (2015) \\
\hline EW0408_85JC & 59.56 & -144.15 & MarineSediment & $\delta^{18} \mathrm{O}$ & http://www.ncdc.noaa.gov/paleo/study/21950 & Praetorius et al. (2015) \\
\hline EW0408-87JC & 58.77 & -144.50 & MarineSediment & Alkenone & wNAm & Praetorius et al. (2020) \\
\hline Farewell Lake & 62.55 & -153.63 & LakeSediment & $\mathrm{Mg} / \mathrm{Ca}$ & http://www.ncdc.noaa.gov/paleo/study/15444 & Hu et al. (1998) \\
\hline Felker Lake & 51.95 & -122.00 & LakeSediment & Diatom & wNAm & Galloway et al. (2011) \\
\hline Ferndale & 34.41 & -95.81 & LakeSediment & Pollen & http://www.ncdc.noaa.gov/paleo/study/27330 & Albert and Wyckoff (1981) \\
\hline Foy Lake & 48.20 & -114.40 & LakeSediment & Diatom & http://www.ncdc.noaa.gov/paleo/study/6188 & Stone and Fritz (2006) \\
\hline Frozen Lake & 49.60 & -121.47 & LakeSediment & Chironomid & http://www.ncdc.noaa.gov/paleo/study/27330 & Rosenberg et al. (2004) \\
\hline GGC19 & 72.16 & -155.51 & MarineSediment & Dinocyst & http://www.ncdc.noaa.gov/paleo/study/15444 & Farmer et al. (2011) \\
\hline Great Basin & 38.00 & -116.50 & Wood & TRW & http://www.ncdc.noaa.gov/paleo/study/17056 & Salzer et al. (2014) \\
\hline Greyling Lake & 61.38 & -145.74 & LakeSediment & TOC & http://www.ncdc.noaa.gov/paleo/study/15444 & McKay and Kaufman (2009) \\
\hline Grutas del Rey Marcos & 15.43 & -90.28 & Speleothem & $\delta^{18} \mathrm{O}$ & http://www.ncdc.noaa.gov/paleo/study/28351 & Winter et al. (2020) \\
\hline Guaymas Basin & 27.48 & -112.07 & MarineSediment & $\delta \mathrm{D}$ & http://www.ncdc.noaa.gov/paleo/study/24890 & Bhattacharya et al. (2018) \\
\hline Guaymas Basin & 27.48 & -112.07 & MarineSediment & $\delta \mathrm{D}$ & http://www.ncdc.noaa.gov/paleo/study/24890 & Bhattacharya et al. (2018) \\
\hline Gulf of Mexico & 27.18 & -91.42 & MarineSediment & Foraminifera & wNAm & Poore et al. (2005) \\
\hline Hail Lake & 60.03 & -129.02 & LakeSediment & Pollen & http://www.ncdc.noaa.gov/paleo/study/15444 & Cwynar and Spear (2007) \\
\hline Hallet Lake & 61.49 & -146.24 & LakeSediment & TOC & http://www.ncdc.noaa.gov/paleo/study/15444 & McKay and Kaufman (2009) \\
\hline Hallet Lake & 61.49 & -146.24 & LakeSediment & $\mathrm{BSi}$ & http://www.ncdc.noaa.gov/paleo/study/15444 & McKay and Kaufman (2009) \\
\hline Hanging Lake & 68.38 & -138.38 & LakeSediment & Pollen & http://www.ncdc.noaa.gov/paleo/study/27330 & Cwynar (1982) \\
\hline Harding Lake & 64.42 & -146.85 & LakeSediment & TOC & http://www.ncdc.noaa.gov/paleo/study/15655 & Finkenbinder et al. (2014) \\
\hline Harding Lake & 64.42 & -146.85 & LakeSediment & MS & http://www.ncdc.noaa.gov/paleo/study/15655 & Finkenbinder et al. (2014) \\
\hline Heal Lake & 48.54 & -123.46 & LakeSediment & Pollen & wNAm & Brown et al. (2006) \\
\hline Hermit Lake & 38.09 & -105.63 & LakeSediment & Pollen & wNAm & R. S. Anderson et al. (2019) \\
\hline Hidden Lake, CA & 38.26 & -119.54 & LakeSediment & Chironomid & http://www.ncdc.noaa.gov/paleo/study/27330 & Potito et al. (2006) \\
\hline
\end{tabular}


Table 1. Continued.

\begin{tabular}{|c|c|c|c|c|c|c|}
\hline Site name & Lat & Long & Archive type & Proxy* & Original data citation (last access: 29 March 2021) & Reference \\
\hline Hidden Lake, $\mathrm{CO}$ & 40.51 & -106.61 & LakeSediment & Stratigraphy & http://www.ncdc.noaa.gov/paleo/study/23077 & Shuman et al. (2009) \\
\hline HLY0501 & 72.69 & -157.52 & MarineSediment & Dinocyst & http://www.ncdc.noaa.gov/paleo/study/15444 & de Vernal et al. (2013) \\
\hline Honeymoon & 64.63 & -138.40 & LakeSediment & Pollen & https://doi.org/10.21233/N33Q7V & Cwynar and Spear (1991) \\
\hline Hudson, AK & 61.90 & -145.67 & LakeSediment & Chironomid & http://www.ncdc.noaa.gov/paleo/study/15444 & Clegg et al. (2011) \\
\hline Hunters Lake & 37.61 & -106.84 & LakeSediment & Pollen & wNAm & R. S. Anderson et al. (2008b) \\
\hline Jellybean Lake & 60.35 & -134.80 & LakeSediment & $\delta^{18} \mathrm{O}$ & http://www.ncdc.noaa.gov/paleo/study/5445 & L. Anderson et al. (2005) \\
\hline Jenny Lake & 43.75 & -110.73 & LakeSediment & TIC & http://www.ncdc.noaa.gov/paleo/study/20128 & Larsen et al. (2016) \\
\hline Jones Lake & 47.05 & -113.14 & LakeSediment & $\delta^{18} \mathrm{O}$ & http://www.ncdc.noaa.gov/paleo/study/23076 & Shapley et al. (2009) \\
\hline Keele & 64.17 & -127.62 & LakeSediment & Pollen & http://www.ncdc.noaa.gov/paleo/study/27330 & Szeicz et al. (1995) \\
\hline Keystone Iron Bog & 38.87 & -107.03 & LakeSediment & Pollen & http://www.ncdc.noaa.gov/paleo/study/27330 & Fall (1985) \\
\hline Kirman Lake & 38.34 & -119.50 & LakeSediment & Diatom & http://dataverse.harvard.edu/dataverse/UCLAGMacDonald & MacDonald et al. (2016) \\
\hline Kite Lake & 39.33 & -106.13 & LakeSediment & Pollen & wNAm & Jiménez-Moreno and Anderson (2013) \\
\hline KNR159_JPC26 & 26.37 & -92.03 & MarineSediment & $\mathrm{Mg} / \mathrm{Ca}$ & http://www.ncdc.noaa.gov/paleo/study/27330 & Antonarakou et al. (2015) \\
\hline KNR159_JPC26 & 26.37 & -92.03 & MarineSediment & $\delta^{18} \mathrm{O}$ & http://www.ncdc.noaa.gov/paleo/study/27330 & Antonarakou et al. (2015) \\
\hline Koksilah River & 48.76 & -123.68 & LakeSediment & Pollen & wNAm & Brown and Schoups (2015) \\
\hline Kurupa Lake & 68.35 & -154.61 & LakeSediment & Chlorophyll & http://www.ncdc.noaa.gov/paleo/study/18995 & Boldt et al. (2015) \\
\hline Kusawa & 60.28 & -136.18 & LakeSediment & $\mathrm{BSi}$ & http://www.ncdc.noaa.gov/paleo/study/15444 & Chakraborty et al. (2010) \\
\hline Lac Meleze & 65.22 & -126.12 & LakeSediment & Pollen & http://www.ncdc.noaa.gov/paleo/study/27330 & MacDonald (1987) \\
\hline Lago Minucua & 17.08 & -97.61 & LakeSediment & MS & wNAm & Goman et al. (2018) \\
\hline Lago Minucua & 17.08 & -97.61 & LakeSediment & Varve & wNAm & Goman et al. (2018) \\
\hline Lago Puerto Arturo & 17.53 & -90.18 & LakeSediment & $\delta^{18} \mathrm{O}$ & wNAm & Wahl et al. (2014) \\
\hline Laguna de Aljojuca & 19.09 & -97.53 & LakeSediment & $\delta^{18} \mathrm{O}$ & http://www.ncdc.noaa.gov/paleo/study/17735 & Bhattacharya et al. (2015) \\
\hline Laguna de Juanacatlan & 20.63 & -104.74 & LakeSediment & $\mathrm{Ti}$ & wNAm & Jones et al. (2015) \\
\hline Lake Elsinore & 33.67 & -117.35 & LakeSediment & $\delta^{18} \mathrm{O}$ & http://www.ncdc.noaa.gov/paleo/study/30232 & Kirby et al. (2019) \\
\hline Lake Elsinore & 33.67 & -117.35 & LakeSediment & Particle size & http://www.ncdc.noaa.gov/paleo/study/30232 & Kirby et al. (2019) \\
\hline Lake of the Woods & 43.48 & -109.89 & LakeSediment & Stratigraphy & wNAm & Pribyl and Shuman (2014) \\
\hline Lake of the Woods & 49.05 & -120.18 & LakeSediment & Chironomid & http://www.ncdc.noaa.gov/paleo/study/27330 & Palmer et al. (2002) \\
\hline Lehman Caves & 39.00 & -114.22 & Speleothem & $\delta^{18} \mathrm{C}$ & http://www.ncdc.noaa.gov/paleo/study/19038 & Steponaitis et al. (2015) \\
\hline Lehman Caves & 39.00 & -114.22 & Speleothem & $\mathrm{Mg} / \mathrm{Ca}$ & http://www.ncdc.noaa.gov/paleo/study/19038 & Steponaitis et al. (2015) \\
\hline Leviathan & 37.89 & -115.58 & Speleothem & $\delta^{18} \mathrm{C}$ & http://www.ncdc.noaa.gov/paleo/study/16517 & Lachniet et al. (2014) \\
\hline Leviathan & 37.89 & -115.58 & Speleothem & $\delta^{18} \mathrm{O}$ & http://www.ncdc.noaa.gov/paleo/study/16517 & Lachniet et al. (2014) \\
\hline Lily & 59.20 & -135.40 & LakeSediment & Pollen & http://www.ncdc.noaa.gov/paleo/study/15444 & Cwynar (1990) \\
\hline Lime Lake & 48.87 & -117.34 & LakeSediment & $\delta^{18} \mathrm{O}$ & http://www.ncdc.noaa.gov/paleo/study/21250 & Steinman et al. (2016) \\
\hline Lime Lake & 48.87 & -117.34 & LakeSediment & $\delta^{18} \mathrm{C}$ & http://www.ncdc.noaa.gov/paleo/study/21250 & Steinman et al. (2016) \\
\hline Little & 44.17 & -123.58 & LakeSediment & Pollen & http://www.ncdc.noaa.gov/paleo/study/27330 & Worona and Whitlock (1995) \\
\hline Little Molas Lake & 37.74 & -107.71 & LakeSediment & Pollen & wNAm & Toney and Anderson (2006) \\
\hline Little Windy & 41.43 & -106.33 & LakeSediment & Stratigraphy & http://www.ncdc.noaa.gov/paleo/study/16096 & Minckley et al. (2012) \\
\hline Logan & 60.58 & -140.50 & GlacierIce & $\delta^{18} \mathrm{O}$ & http://www.ncdc.noaa.gov/paleo/study/15444 & Fisher et al. (2008) \\
\hline Lone Fox Lake & 56.72 & -119.72 & LakeSediment & Pollen & http://www.ncdc.noaa.gov/paleo/study/27330 & MacDonald and Cwynar (1985) \\
\hline Lone Spruce & 60.01 & -159.14 & LakeSediment & $\mathrm{BSi}$ & http://www.ncdc.noaa.gov/paleo/study/15444 & Kaufman et al. (2012) \\
\hline Louise Pond & 52.95 & -131.76 & LakeSediment & Pollen & http://www.ncdc.noaa.gov/paleo/study/27330 & Pellatt and Mathewes (1994) \\
\hline Lowder Creek Bog & 37.66 & -112.77 & Peat & Pollen & wNAm & R. S. Anderson et al. (1999) \\
\hline Lower Bear Lake & 34.20 & -116.90 & LakeSediment & TOC & http://www.ncdc.noaa.gov/paleo/study/13215 & Kirby et al. (2012) \\
\hline Lower Bear Lake & 34.20 & -116.90 & LakeSediment & $\mathrm{C} / \mathrm{N}$ & http://www.ncdc.noaa.gov/paleo/study/13215 & Kirby et al. (2012) \\
\hline M Lake & 68.27 & -133.47 & LakeSediment & Pollen & http://www.ncdc.noaa.gov/paleo/study/27330 & Ritchie (1977) \\
\hline Macal Chasm & 16.88 & -89.11 & Speleothem & $\delta^{18} \mathrm{C}$ & http://www.ncdc.noaa.gov/paleo/study/20506 & Akers et al. (2016) \\
\hline Macal Chasm & 16.88 & -89.11 & Speleothem & $\delta^{18} \mathrm{O}$ & http://www.ncdc.noaa.gov/paleo/study/20506 & Akers et al. (2016) \\
\hline Macal Chasm & 16.88 & -89.11 & Speleothem & Reflectance & http://www.ncdc.noaa.gov/paleo/study/20506 & Akers et al. (2016) \\
\hline Marcella & 60.07 & -133.81 & LakeSediment & $\delta^{18} \mathrm{O}$ & http://www.ncdc.noaa.gov/paleo/study/6066 & L. Anderson et al. (2007) \\
\hline Marion & 49.31 & -122.55 & LakeSediment & Pollen & wNAm & Mathewes (1973) \\
\hline Marshall Lake & 40.68 & -110.87 & LakeSediment & $\mathrm{Ca} / \mathrm{Ti}$ & wNAm & Munroe et al. (2020) \\
\hline MD02_2503 & 34.39 & -120.04 & MarineSediment & $\delta^{18} \mathrm{O}$ & http://www.ncdc.noaa.gov/paleo/study/5582 & Hill et al. (2006) \\
\hline MD02_2515 & 27.48 & -112.07 & MarineSediment & Alkenone & https://doi.org/10.1594/PANGAEA.861260 & McClymont et al. (2012) \\
\hline MD02_2515 & 27.48 & -112.07 & MarineSediment & GDGT & https://doi.org/10.1594/PANGAEA.861260 & McClymont et al. (2012) \\
\hline MD02-2499 & 41.65 & -124.94 & MarineSediment & Diatom & http://www.ncdc.noaa.gov/paleo/study/24150 & Lopes and Mix (2018) \\
\hline Meli Lake & 68.68 & -149.08 & LakeSediment & $\delta^{18} \mathrm{O}$ & http://www.ncdc.noaa.gov/paleo/study/5469 & L. Anderson et al. (2001) \\
\hline Mexican Marin & 22.23 & -107.05 & MarineSediment & $\delta \mathrm{D}$ & http://www.ncdc.noaa.gov/paleo/study/24890 & Bhattacharya et al. (2018) \\
\hline Mica Lake & 60.95 & -148.15 & LakeSediment & $\delta^{18} \mathrm{O}$ & http://www.ncdc.noaa.gov/paleo/study/6202 & Schiff et al. (2009) \\
\hline Midden Cluster 1 & 37.90 & -110.13 & Midden & Macrofossils & http://geochange.er.usgs.gov/midden/ & Harbert and Nixon (2018) \\
\hline Midden Cluster 2 & 36.38 & -115.19 & Midden & Macrofossils & http://geochange.er.usgs.gov/midden/ & Harbert and Nixon (2018) \\
\hline Midden Cluster 3 & 36.06 & -108.08 & Midden & Macrofossils & http://geochange.er.usgs.gov/midden/ & Harbert and Nixon (2018) \\
\hline Midden Cluster 4 & 43.65 & -112.75 & Midden & Macrofossils & http://geochange.er.usgs.gov/midden/ & Harbert and Nixon (2018) \\
\hline Midden Cluster 5 & 32.47 & -106.02 & Midden & Macrofossils & http://geochange.er.usgs.gov/midden/ & Harbert and Nixon (2018) \\
\hline Midden Cluster 6 & 32.47 & -106.02 & Midden & Macrofossils & http://geochange.er.usgs.gov/midden/ & Harbert and Nixon (2018) \\
\hline Midden Cluster 7 & 34.15 & -116.00 & Midden & Macrofossils & http://geochange.er.usgs.gov/midden/ & Harbert and Nixon (2018) \\
\hline Midden Cluster 8 & 32.31 & -109.10 & Midden & Macrofossils & http://geochange.er.usgs.gov/midden/ & Harbert and Nixon (2018) \\
\hline Midden Cluster 9 & 31.64 & -115.55 & Midden & Macrofossils & http://geochange.er.usgs.gov/midden/ & Harbert and Nixon (2018) \\
\hline Minnetonka Cave & 42.09 & -111.52 & Speleothem & $\delta^{18} \mathrm{C}$ & http://www.ncdc.noaa.gov/paleo/study/23097 & Lundeen et al. (2013) \\
\hline Minnetonka Cave & 42.09 & -111.52 & Speleothem & $\delta^{18} \mathrm{O}$ & http://www.ncdc.noaa.gov/paleo/study/23097 & Lundeen et al. (2013) \\
\hline Moose Lake & 61.37 & -143.60 & LakeSediment & Chironomid & http://www.ncdc.noaa.gov/paleo/study/15444 & Clegg et al. (2010) \\
\hline Morris Pond & 37.67 & -112.77 & LakeSediment & Pollen & wNAm & Morris et al. (2013) \\
\hline Mv0811-14JC & 34.30 & -120.00 & MarineSediment & Stratigraphy & wNAm & Du et al. (2018) \\
\hline MV99_PC14 & 25.20 & -112.72 & MarineSediment & $\mathrm{Mg} / \mathrm{Ca}$ & http://www.ncdc.noaa.gov/paleo/study/10415 & Marchitto et al. (2010) \\
\hline MV99-GC31 & 23.47 & -111.60 & MarineSediment & $\mathrm{BSi}$ & https://doi.org/10.1594/PANGAEA.824830 & Barron et al. (2012) \\
\hline MV99-GC41/PC14 & 25.20 & -112.72 & MarineSediment & Particle size & https://doi.org/10.1594/PANGAEA.896898 & Arellano-Torres et al. (2019) \\
\hline Natural Bridge Caverns & 29.69 & -98.34 & Speleothem & $\mathrm{Sr}$ & wNAm & Wong et al. (2015) \\
\hline Nevada Climate Division 3 & 37.80 & -115.80 & Wood & TRW & http://www.ncdc.noaa.gov/paleo/study/6384 & Hughes and Graumlich (1996) \\
\hline North Crater Lake & 49.07 & -120.02 & LakeSediment & Chironomid & http://www.ncdc.noaa.gov/paleo/study/27330 & Palmer et al. (2002) \\
\hline ODP_167_1019C & 41.68 & -124.93 & MarineSediment & Alkenone & https://doi.org/10.1594/PANGAEA.841946 & Barron et al. (2003b) \\
\hline
\end{tabular}


Table 1. Continued.

\begin{tabular}{|c|c|c|c|c|c|c|}
\hline Site name & Lat & Long & Archive type & Proxy* & Original data citation (last access: 29 March 2021) & Reference \\
\hline ODP1019 & 41.68 & -124.93 & MarineSediment & Diatom & http://www.ncdc.noaa.gov/paleo/study/24150 & Lopes and Mix (2018) \\
\hline ODP1019 & 41.68 & -124.93 & MarineSediment & $\mathrm{CaCO}_{3}$ & http://www.ncdc.noaa.gov/paleo/study/5867 & Barron et al. (2003b) \\
\hline ODP1019 & 41.68 & -124.93 & MarineSediment & Pollen & http://www.ncdc.noaa.gov/paleo/study/5867 & Barron et al. (2003b) \\
\hline Oregon Caves & 42.08 & -123.42 & Speleothem & $\delta^{18} \mathrm{C}$ & http://www.ncdc.noaa.gov/paleo/study/13543 & Ersek et al. (2012) \\
\hline Oregon Caves & 42.08 & -123.42 & Speleothem & $\delta^{18} \mathrm{O}$ & http://www.ncdc.noaa.gov/paleo/study/13543 & Ersek et al. (2012) \\
\hline Oro Lake & 49.78 & -105.35 & LakeSediment & Diatom & http://www.ncdc.noaa.gov/paleo/study/23073 & Michels et al. (2007) \\
\hline Owens Lake & 36.44 & -117.97 & LakeSediment & $\delta^{18} \mathrm{O}$ & http://www.ncdc.noaa.gov/paleo/study/5472 & Benson et al. (2002) \\
\hline P1B3 & 73.68 & -162.66 & MarineSediment & Dinocyst & http://www.ncdc.noaa.gov/paleo/study/15444 & de Vernal et al. (2005) \\
\hline Paradise & 54.69 & -122.62 & LakeSediment & $\delta^{18} \mathrm{O}$ & http://www.ncdc.noaa.gov/paleo/study/21250 & Steinman et al. (2016) \\
\hline Paradise & 54.69 & -122.62 & LakeSediment & $\delta^{18} \mathrm{C}$ & http://www.ncdc.noaa.gov/paleo/study/21250 & Steinman et al. (2016) \\
\hline Park Pond 1 & 43.47 & -109.96 & LakeSediment & Pollen & http://www.ncdc.noaa.gov/paleo/study/27330 & Lynch (1998) \\
\hline Pink Panther & 32.08 & -105.17 & Speleothem & $\delta^{18} \mathrm{O}$ & http://www.ncdc.noaa.gov/paleo/study/9739 & Asmerom et al. (2007) \\
\hline Pixie & 48.60 & -124.20 & LakeSediment & Pollen & http://www.ncdc.noaa.gov/paleo/study/27330 & Brown and Hebda (2002) \\
\hline Pixie Lake & 48.60 & -124.20 & LakeSediment & Pollen & wNAm & Brown et al. (2006) \\
\hline Posy & 37.94 & -111.70 & LakeSediment & Pollen & http://www.ncdc.noaa.gov/paleo/study/27330 & Shafer (1989) \\
\hline PS1410-06GC & 37.33 & -123.40 & MarineSediment & Pollen & wNAm & Barron et al. (2018) \\
\hline PS1410-06GC & 37.33 & -123.40 & MarineSediment & $\mathrm{BSi}$ & wNAm & Barron et al. (2018) \\
\hline Pyramid Lake & 40.07 & -119.58 & LakeSediment & $\delta^{18} \mathrm{O}$ & http://www.ncdc.noaa.gov/paleo/study/5472 & Benson et al. (2002) \\
\hline Quartz & 64.21 & -145.81 & LakeSediment & Chironomid & http://www.ncdc.noaa.gov/paleo/study/15444 & Wooller et al. (2012) \\
\hline Rainbow & 60.72 & -150.80 & LakeSediment & Chironomid & http://www.ncdc.noaa.gov/paleo/study/15444 & Clegg et al. (2011) \\
\hline Rainbow Lake & 44.94 & -109.50 & LakeSediment & Stratigraphy & wNAm & Shuman and Marsicek (2016) \\
\hline Ranger & 67.15 & -153.65 & LakeSediment & Pollen & http://www.ncdc.noaa.gov/paleo/study/15444| & Brubaker et al. (1983) \\
\hline Rantin Lake & 60.03 & -129.03 & LakeSediment & $\mathrm{CaCO}_{3}$ & http://www.ncdc.noaa.gov/paleo/study/13095 & Pompeani et al. (2012) \\
\hline Rapid & 42.73 & -109.19 & LakeSediment & Pollen & http://www.ncdc.noaa.gov/paleo/study/27330 & Fall (1988) \\
\hline $\mathrm{RC} 12-10$ & 23.00 & -95.53 & MarineSediment & Foraminifera & http://www.ncdc.noaa.gov/paleo/study/27330 & Poore et al. (2003) \\
\hline Red Rock & 40.08 & -105.54 & LakeSediment & Pollen & http://www.ncdc.noaa.gov/paleo/study/27330 & Maher (1972) \\
\hline Rhamnus Lake & 48.63 & -123.72 & LakeSediment & Pollen & wNAm & Brown et al. (2006) \\
\hline San Juan River Discharge & 48.58 & -124.31 & LakeSediment & Pollen & wNAm & Brown and Schoups (2015) \\
\hline Schellings Bog & 40.28 & -123.36 & LakeSediment & Pollen & wNAm & Barron et al. (2003a) \\
\hline Screaming Lynx Lake & 66.07 & -145.40 & LakeSediment & Chironomid & http://www.ncdc.noaa.gov/paleo/study/15444 & Clegg et al. (2011) \\
\hline Silver Lake & 35.37 & -116.14 & LakeSediment & Particle size & http://www.ncdc.noaa.gov/paleo/study/20106 & Kirby et al. (2015) \\
\hline Silver Lake & 35.37 & -116.14 & LakeSediment & $\mathrm{C} / \mathrm{N}$ & http://www.ncdc.noaa.gov/paleo/study/20106 & Kirby et al. (2015) \\
\hline Southern California & 33.77 & -116.66 & Peat & Pollen & http://www.ncdc.noaa.gov/paleo/study/27330 & Ohlwein and Wahl (2012) \\
\hline Station 803 & 70.63 & -135.88 & MarineSediment & Dinocyst & http://www.ncdc.noaa.gov/paleo/study/27910 & Bringué and Rochon (2012) \\
\hline Stella Lake & 39.01 & -114.32 & LakeSediment & Chironomid & http://www.ncdc.noaa.gov/paleo/study/27330 & Reinemann et al. (2009) \\
\hline Stewart Bog & 35.83 & -105.72 & Peat & Pollen & wNAm & Jiménez-Moreno et al. (2008) \\
\hline Stowell Lake & 48.78 & -123.44 & LakeSediment & Chironomid & http://www.ncdc.noaa.gov/paleo/study/27330 & Lemmen and Lacourse (2018) \\
\hline Swan Lake & 42.16 & -99.03 & LakeSediment & Diatom & wNAm & Schmieder et al. (2011) \\
\hline Swasey Lake & 40.67 & -110.47 & LakeSediment & $\mathrm{Ca} / \mathrm{Ti}$ & wNAm & Munroe et al. (2020) \\
\hline Takahula & 67.35 & -153.67 & LakeSediment & $\delta^{18} \mathrm{O}$ & http://www.ncdc.noaa.gov/paleo/study/8663 & Clegg and $\mathrm{Hu}(2010)$ \\
\hline Tangled Up Lake & 67.67 & -149.08 & LakeSediment & $\delta^{18} \mathrm{O}$ & http://www.ncdc.noaa.gov/paleo/study/5469 & L. Anderson et al. (2001) \\
\hline Taylor Lake & 40.79 & -110.09 & LakeSediment & $\mathrm{Ca} / \mathrm{Ti}$ & wNAm & Munroe et al. (2020) \\
\hline Tiago Lake & 40.58 & -106.61 & LakeSediment & Pollen & wNAm & Jiménez-Moreno et al. (2011) \\
\hline TN062-0550 & 40.87 & -124.57 & MarineSediment & Pollen & http://www.ncdc.noaa.gov/paleo/study/27330 & Barron et al. (2018) \\
\hline TN062-0550 & 40.87 & -124.57 & MarineSediment & $\mathrm{BSi}$ & http://www.ncdc.noaa.gov/paleo/study/27330 & Addison et al. (2018) \\
\hline TN062-0550 & 40.87 & -124.57 & MarineSediment & $\delta^{18} \mathrm{C}$ & http://www.ncdc.noaa.gov/paleo/study/27330 & Addison et al. (2018) \\
\hline TN062-0550 & 40.87 & -124.57 & MarineSediment & $\delta^{15} \mathrm{~N}$ & http://www.ncdc.noaa.gov/paleo/study/27330 & Addison et al. (2018) \\
\hline Trout Lake & 68.83 & -138.75 & LakeSediment & Chironomid & http://www.ncdc.noaa.gov/paleo/study/15444 & Irvine et al. (2012) \\
\hline Upper Big Creek Lake & 40.91 & -106.62 & LakeSediment & Stratigraphy & wNAm & Shuman et al. (2015) \\
\hline Upper Fly & 61.07 & -138.09 & LakeSediment & Pollen & http://www.ncdc.noaa.gov/paleo/study/15444 & Bunbury and Gajewski (2009) \\
\hline Upper Pinto Fen & 53.58 & -118.02 & Peat & DBD & http://www.ncdc.noaa.gov/paleo/study/13665 & Yu et al. (2003) \\
\hline W8709-13PC & 42.12 & -125.75 & MarineSediment & Diatom & http://www.ncdc.noaa.gov/paleo/study/24150 & Lopes and Mix (2018) \\
\hline WA01 & 61.24 & -136.93 & LakeSediment & TOC & http://www.ncdc.noaa.gov/paleo/study/18435 & Rainville and Gajewski (2013) \\
\hline Waskey Lake & 59.88 & -159.21 & LakeSediment & TOC & http://www.ncdc.noaa.gov/paleo/study/15444 & Levy et al. (2004) \\
\hline Windy Lake & 49.81 & -117.88 & LakeSediment & Chironomid & http://www.ncdc.noaa.gov/paleo/study/27330 & Chase et al. (2008) \\
\hline Wolverine Lake & 67.10 & -158.91 & LakeSediment & MAR & http://www.ncdc.noaa.gov/paleo/study/23070 & Mann et al. (2002) \\
\hline Yellow Lake & 39.65 & -107.35 & LakeSediment & $\delta^{18} \mathrm{O}$ & http://www.ncdc.noaa.gov/paleo/study/13120 & L. Anderson (2012) \\
\hline
\end{tabular}

These primary age controls can be used to recalculate the age models for all of the ${ }^{14} \mathrm{C}$-based sedimentary sequences and U-series-based speleothems using a systematic approach to addressing age uncertainty.

\subsection{Uncertainties}

A variety of approaches have been used to characterize uncertainties in paleoclimate variables, and there is no standard procedure for either calculating or reporting uncertainties (Sweeney et al., 2018). Generally, calibration and other uncertainties are large relative to the small amplitude of most
Holocene climate change, but these uncertainties are less important when investigating the relative magnitude of climate changes rather than the absolute value of a climate variable. Uncertainty arising from differences among records can be explored using a bootstrapped sampling with a replacement approach (e.g., Boos, 2003; Routson et al., 2019a); however, these ranges reflect a combination of record-level uncertainty and regional climate heterogeneity. In this database we are following other syntheses (Kaufman et al., 2020b; Marcott et al., 2013; Routson et al., 2019a) by applying a single uncertainty estimate for each proxy type (Supplement Table S1). 


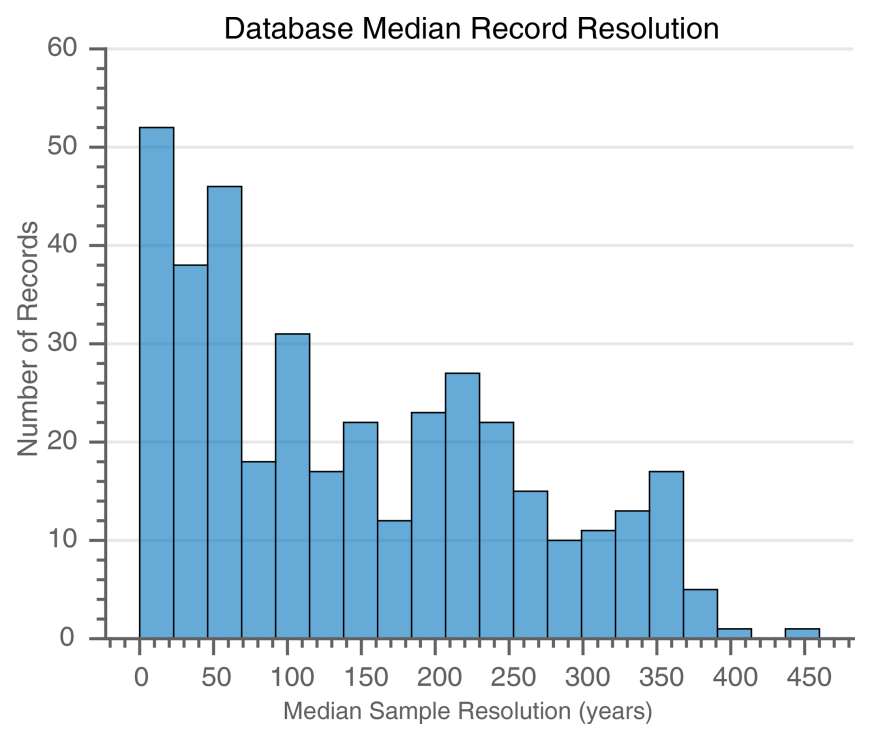

Figure 2. Median sample resolution for all records in the database (20-year intervals).

Proxy-specific uncertainties for temperature records follow Kaufman et al. (2020b), as did our approach for calculating uncertainty estimates for the hydroclimate records. For the calibrated hydroclimate records (primarily pollen based), we have calculated average RMSE values from the following references within or adjacent to the study region (Brown et al., 2006; Brown and Schoups, 2015, 2019; Harbert and Nixon, 2018; Marsicek et al., 2013). For the 163 uncalibrated records we have estimated the error as \pm 1 SD (standard deviation) of the Holocene values.

\subsection{Summarizing major trends}

Recognizing major climatological differences across the study domain (spanning from tropical Mexico to Arctic Alaska), we have summarized some dominant patterns in the database including climate variables (temperature and hydroclimate), proxy group, and season. Dominant temperature and hydroclimate patterns by proxy group as specified in proxy general in Supplement Table S1 were evaluated (Fig. 3). Only proxy groups with more than 10 records were considered. The records were screened by season to include one record per site ("season general" for "annual" or "summer only" or "winter only"). Records were then binned to 500-year resolution by averaging data points within respective intervals, normalized to a mean of zero and 1 SD variance ( $z$ scores), and composited using the median to minimize the influence of outliers. Dominant temperature proxies include chironomids $(n=15)$, biophysical $(n=17)$, pollen $(n=130)$, and isotopes $(n=14)$. Chironomids show peak warmth in the Early Holocene (ca. $10 \mathrm{ka}$ ), followed by a Holocene cooling trend. Biophysical records have more variability, with peak warming at ca. $7 \mathrm{ka}$. Pollen records show relatively low Holocene variability, with peak warming at ca. $6 \mathrm{ka}$. Isotopes have the highest Holocene variability and the lowest sample depth and show two intervals of warming (ca. 9 and $4 \mathrm{ka}$ ). Dominant hydroclimate proxies include other microfossils $(n=11)$, biophysical records $(n=46)$, pollen $(n=57)$, and isotopes $(n=35)$. Other microfossils show variable Holocene conditions, with the wettest period in the Early Holocene. This interval however, has very low sample depth. Biophysical records show only small Holocene hydroclimate changes. Pollen records show a strong Holocene wetting trend, whereas isotope records show variable conditions.

Temperature and hydroclimate trends were compared by summer, winter, and annual seasons (Fig. 4). The records were binned to 500 -year resolution by averaging data points within respective intervals and normalized to a mean of zero and $1 \mathrm{SD}$ variance ( $z$ scores). Records were then averaged into equal-area $\left(127525 \mathrm{~km}^{2}\right)$ grids following Routson et al. (2019a). The grids were then combined into a single composite using the median. The most recent 500 -year bin was then subtracted, registering the present end to zero. This was done to help compare the seasonal Holocene evolutions. In the Early to Middle Holocene (ca 12 to $6 \mathrm{ka}$ ), summertime and annual temperatures warmed faster than wintertime temperatures, consistent with Northern Hemisphere seasonal insolation forcing (Berger and Loutre, 1991). Temperatures in all seasons show a cooling pattern from ca. $6 \mathrm{ka}$ to the present. Hydroclimate composites show a Holocene-length wetting trend in all seasons, with the largest trend in wintertime.

\section{Code and data availability}

The database is available for download at https://doi.org/10.6084/m9.figshare.12863843.v1 (Routson and McKay, 2020), with serializations for MAT$\mathrm{LAB}$ and $\mathrm{R}$. We recommend accessing the database through the WDS-NOAA landing page where any subsequent versions will be made available: https://www.ncdc.noaa.gov/paleo/study/30535 (last access: 8 April 2021). Supplement Table S1 lists the essential metadata. Data can also be viewed and accessed at http://lipdverse.org/wNAm/1_0_0/ (last access: 29 March 2021). Code, including basic functions for analyzing LiPD files in three programming languages, is available on GitHub (https://github.com/nickmckay/ LiPD-utilities, last access: 15 April 2021) and Zenodo (https://doi.org/10.5281/zenodo.1256889, Heiser et al., 2018).

\section{Use and limitations}

The machine-readable database includes multiple parameters for searching and screening records. The data compilation will form the foundation of new analyses of Holocene 
Temperature by proxy type
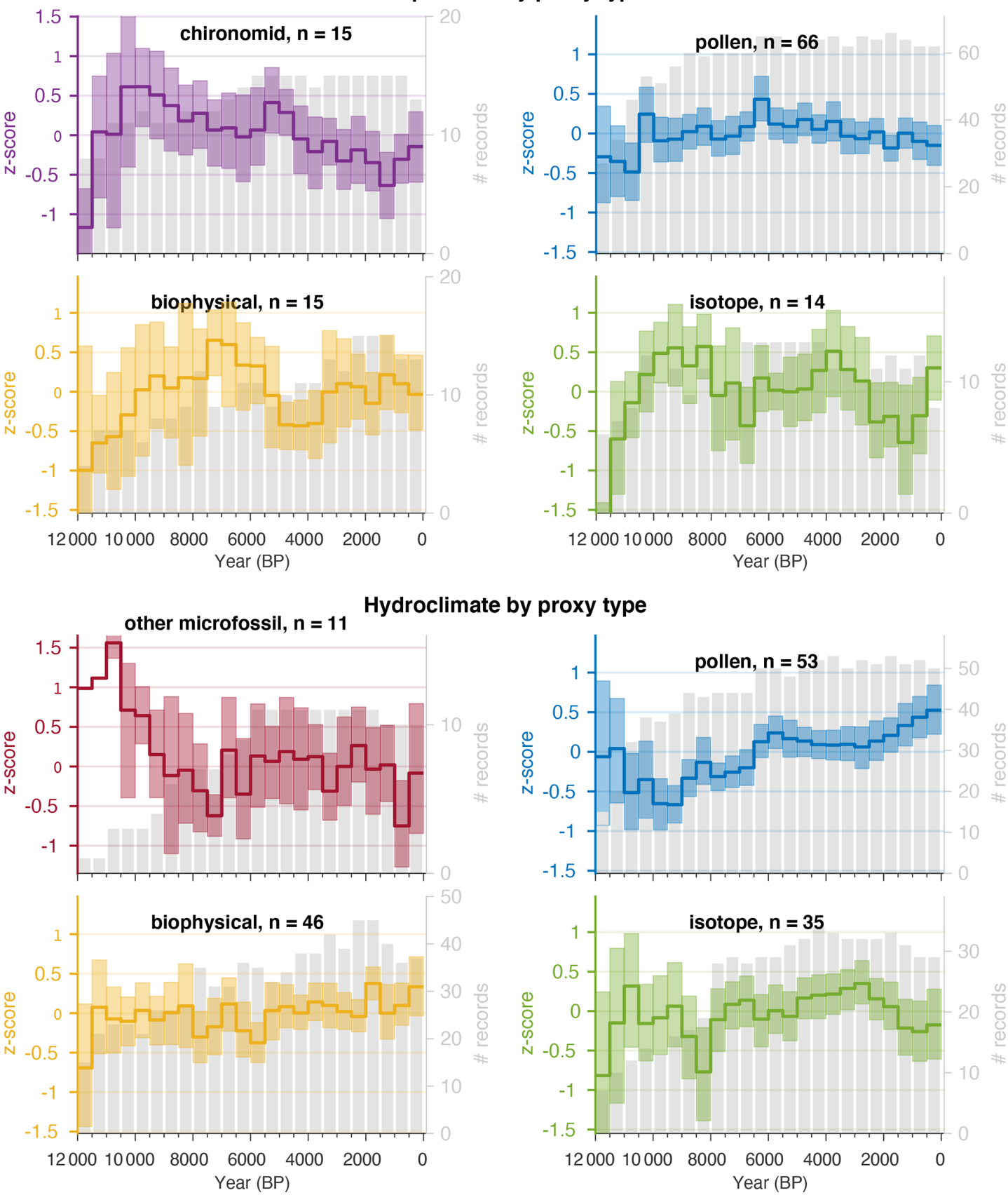

Figure 3. Temperature (top) and hydroclimate (bottom) composites by dominant proxy types (proxy general in Supplement Table S1). Only proxy types with $n>10$ are shown. The composites are produced from normalized (units of standard deviation) records to include both calibrated and uncalibrated time series. Records have been filtered by seasonality (season general for annual, summer only, and winter only), to include one record per site. Shading shows the $95 \%$ bootstrapped confidence interval on the estimate of the mean over 1000 (sampling with replacement) iterations. Gray bars show the number of records contributing to each 500-year bin.

climate variability in western North America and will help identify future research priorities, including data-sparse regions. The 381 records in this database will enable studies of Holocene climate on centennial to multi-millennial timescales. At finer timescales, the number of records with sufficient resolution and geochronological control is more limited. For example, 170 records have a median sampling resolution of better than 100 years, and only 26 sites have resolution finer than 10 years. The accuracy and precision of age control can also limit inferences involving correlations 

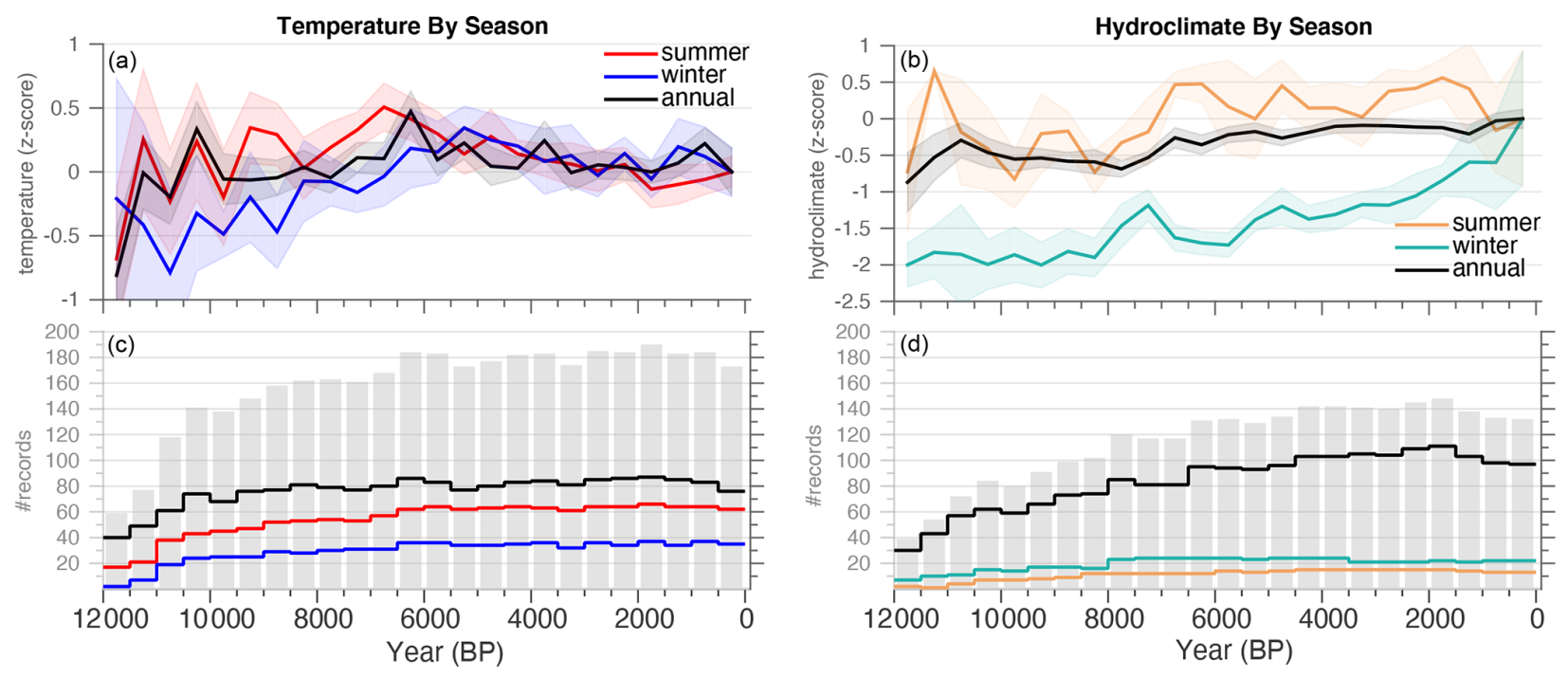

Figure 4. Comparison of seasonal temperature (a, c) and hydroclimate (b, d) composites. The composites are produced from binned (500year bins) and normalized (units of standard deviation) records averaged on an equal area grid. The most recent bin has been registered to zero to help compare the Holocene trends with respect to preindustrial conditions. Both calibrated and uncalibrated time series are included. Shading shows the bootstrapped confidence interval of 1 standard deviation on the estimate of the mean over 1000 (sampling with replacement) iterations. Gray bars (c, d) show the total number of records (all seasons) in each 500-year bin, whereas the time series (c, d) show the number or records contributing to each composite by color.

and spectral properties of the time series. The availability of the raw chronology data for each record in this database allows users to quantify and incorporate aspects of chronologic uncertainty into their analyses.

This database represents a concerted effort to generate a comprehensive data product but is an ongoing effort, with newly published records continuing to be added. Some published records that meet the criteria might have been inadvertently overlooked. Readers who know of missing datasets or who find errors in this version are asked to contact one of the authors so that future versions of the database will be more complete and accurate. Rather than issuing errata to this publication, errors and additions will be included in subsequent versions of the database.

Supplement. The supplement related to this article is available online at: https://doi.org/10.5194/essd-13-1613-2021-supplement.

Author contributions. CCR led the project, data collection, and data formatting. CCR, DSK, MPE, NPM, MEK, JPM, FSU, MSL, SHA, JRB, MFG, SEM, KJB, JMG, SCF, GS, JRR, JLM, DBW, RSA, BNS, JSM, BSC, and GJM contributed and certified data. CCR and MPE analyzed the database and produced the figures. NPM built the data infrastructure and performed data processing. CCR, DSK, and SHA did quality control, term standardization, and database cleaning. CCR and DSK wrote the paper with contributions from the other authors.
Competing interests. The authors declare that they have no conflict of interest.

Acknowledgements. We thank the USGS John Wesley Powell Center for Analysis and Synthesis, which hosted a meeting that led to this synthesis effort. Any use of trade, firm, or product names is for descriptive purposes only and does not imply endorsement by the US government. We thank the original data generators who made their data available for reuse, and we acknowledge the data repositories for safeguarding these assets.

Financial support. This research has been supported by the Directorate for Geosciences of the National Science Foundation (grant nos. AGS-1602105 and AGS-1903548).

Review statement. This paper was edited by Thomas Blunier and reviewed by Jessie Woodbridge and one anonymous referee.

\section{References}

Adams, D. K. and Comrie, A. C.: The North American Monsoon, B. Am. Meteorol. Soc., 78, 2197-2213, https://doi.org/10.1175/15200477(1997)078<2197:TNAM>2.0.CO;2, 1997.

Addison, J. A., Barron, J., Finney, B., Kusler, J., Bukry, D., Heusser, L. E., and Alexander, C. R.: A Holocene record of ocean productivity and upwelling from the north- 
ern California continental slope, Quatern. Int., 469, 96-108, https://doi.org/10.1016/j.quaint.2017.02.021, 2018.

Akers, P. D., Brook, G. A., Railsback, L. B., Liang, F., Iannone, G., Webster, J. W., Reeder, P. P., Cheng, H., and Edwards, R. L.: An extended and higher-resolution record of climate and land use from stalagmite MC01 from Macal Chasm, Belize, revealing connections between major dry events, overall climate variability, and Maya sociopolitical changes, Palaeogeogr. Palaeocl., 459, 268-288, https://doi.org/10.1016/j.palaeo.2016.07.007, 2016.

Albani, S., Mahowald, N. M., Winckler, G., Anderson, R. F., Bradtmiller, L. I., Delmonte, B., François, R., Goman, M., Heavens, N. G., Hesse, P. P., Hovan, S. A., Kang, S. G., Kohfeld, K. E., Lu, H., Maggi, V., Mason, J. A., Mayewski, P. A., McGee, D., Miao, X., Otto-Bliesner, B. L., Perry, A. T., Pourmand, A., Roberts, H. M., Rosenbloom, N., Stevens, T., and Sun, J.: Twelve thousand years of dust: the Holocene global dust cycle constrained by natural archives, Clim. Past, 11, 869-903, https://doi.org/10.5194/cp-11869-2015, 2015.

Albert, L. E. and Wyckoff, D. G.: Ferndale Bog and Natural Lake: Five thousand years of environmental change in southeastern Oklahoma, Oklahoma Archaeological Survey, Norman, USA, 1981.

Anderson, L.: Holocene record of precipitation seasonality from lake calcite $\delta^{18} \mathrm{O}$ in the central Rocky Mountains, United States, Geology, 39, 211-214, https://doi.org/10.1130/G31575.1, 2011.

Anderson, L.: Rocky Mountain hydroclimate: Holocene variability and the role of insolation, ENSO, and the North American Monsoon, Global Planet. Change, 92/93, 198-208, https://doi.org/10.1016/j.gloplacha.2012.05.012, 2012.

Anderson, L., Abbott, M. B., and Finney, B. P.: Holocene climate inferred from oxygen isotope ratios in lake sediments, Central Brooks Range, Alaska, Quaternary Res., 55, 313-321, https://doi.org/10.1006/qres.2001.2219, 2001.

Anderson, L., Abbott, M. B., Finney, B. P., and Burns, S. J.: Regional atmospheric circulation change in the North Pacific during the Holocene inferred from lacustrine carbonate oxygen isotopes, Yukon Territory, Canada, Quaternary Res., 64, 21-35, https://doi.org/10.1016/j.yqres.2005.03.005, 2005.

Anderson, L., Abbott, M. B., Finney, B. P., and Burns, S. J.: Late Holocene moisture balance variability in the southwest Yukon Territory, Canada, Quaternary Sci. Rev., 26, 130-141, https://doi.org/10.1016/j.quascirev.2006.04.011, 2007.

Anderson, R. S., Hasbargen, J., Koehler, P. A., and Feiler, E. J.: Late Wisconsin and Holocene subalpine forests of the Markagunt Plateau of Utah, southwestern Colorado Plateau, USA, Arct. Antarct. Alp. Res., 31, 366-378, https://doi.org/10.1080/15230430.1999.12003321, 1999.

Anderson, R. S., Jass, R. B., Toney, J. L., Allen, C. D., CisnerosDozal, L. M., Hess, M., Heikoop, J., and Fessenden, J.: Development of the mixed conifer forest in northern New Mexico and its relationship to Holocene environmental change, Quaternary Res., 69, 263-275, https://doi.org/10.1016/j.yqres.2007.12.002, 2008a.

Anderson, R. S., Allen, C. D., Toney, J. L., Jass, R. B., and Bair, A. N.: Holocene vegetation and fire regimes in subalpine and mixed conifer forests, southern Rocky Mountains, USA, Int. J. Wildland Fire, 17, 96-114, https://doi.org/10.1071/WF07028, $2008 \mathrm{~b}$.

Anderson, R. S., Soltow, H. R., and Jiménez-Moreno, G.: Postglacial environmental change of a high-elevation for- est, Sangre de Cristo Mountains of south-central Colorado, in: From Saline to Freshwater: The Diversity of Western Lakes in Space and Time, edited by: Starratt, S. W. and Rosen, M. R., Geological Society of America Special Papers, https://doi.org/10.1130/2018.2536(13), 2019.

Anil: digitize2.m, MATLAB Central File Exchange, available at: https://www.mathworks.com/matlabcentral/fileexchange/ 928-digitize2-m (last access: 29 March 2021), 2020.

Antonarakou, A., Kontakiotis, G., Mortyn, P. G., Drinia, H., Sprovieri, M., Besiou, E., and Tripsanas, E.: Biotic and geochemical $\left(\delta^{18} \mathrm{O}, \delta^{13} \mathrm{C}, \mathrm{Mg} / \mathrm{Ca}, \mathrm{Ba} / \mathrm{Ca}\right)$ responses of Globigerinoides ruber morphotypes to upper water column variations during the last deglaciation, Gulf of Mexico, Geochim. Cosmochim. Ac., 170, 69-93, https://doi.org/10.1016/j.gca.2015.08.003, 2015.

Arcusa, S. H., McKay, N. P., Routson, C. C., and Munoz, S. E.: Dust-drought interactions over the last 15,000 years: A network of lake sediment records from the San Juan Mountains, Colorado, Holocene, 30, 559-574, https://doi.org/10.1177/0959683619875192, 2020.

Arellano-Torres, E., Álvarez-Covelli, C., Kasper-Zubillaga, J. J., and Lozano-García, M. S.: A 14-ka record of dust input and phytoplankton regime changes in the subtropical NE Pacific: Oceanic and terrestrial processes linked by teleconnections at suborbital scales, Paleoceanography and Paleoclimatology, 34, 35-53, https://doi.org/10.1029/2018PA003479, 2019.

Asmerom, Y., Polyak, V., Burns, S., and Rassmussen, J.: Solar forcing of Holocene climate: New insights from a speleothem record, southwestern United States, Geology, 35, 14, https://doi.org/10.1130/G22865A.1, 2007.

Barnosky, C. W.: Late Quaternary vegetation in the southwestern Columbia Basin, Washington, Quaternary Res., 23, 109-122, https://doi.org/10.1016/0033-5894(85)90075-4, 1985a.

Barnosky, C. W.: Late Quaternary vegetation near Battle Ground Lake, southern Puget Trough, Washington, Geol. Soc. Am. Bull., 96, 263-271, https://doi.org/10.1130/00167606(1985)96<263:LQVNBG>2.0.CO;2, 1985b.

Barron, J. A., Heusser, L. E., and Alexander, C.: High resolution climate of the past 3,500 years of coastal northernmost California, in: Proceedings of the Twentieth Annual Pacific Climate Workshop, 13-22, 2003a.

Barron, J. A., Heusser, L., Herbert, T., and Lyle, M.: Highresolution climatic evolution of coastal northern California during the past 16,000 years, Paleoceanography, 18, 1020, https://doi.org/10.1029/2002PA000768, 2003b.

Barron, J. A., Bukry, D., and Bischoff, J. L.: High resolution paleoceanography of the Guaymas Basin, Gulf of California, during the past 15000 years, Mar. Micropaleontol., 50, 185-207, https://doi.org/10.1016/S0377-8398(03)00071-9, 2004.

Barron, J. A., Metcalfe, S. E., and Addison, J. A.: Response of the North American monsoon to regional changes in ocean surface temperature, Paleoceanography, 27, PA3206, https://doi.org/10.1029/2011PA002235, 2012.

Barron, J. A., Bukry, D., Heusser, L. E., Addison, J. A., and Alexander, C. R.: High-resolution climate of the past $\sim 7300$ years of coastal northernmost California: Results from diatoms, silicoflagellates, and pollen, Quatern. Int., 469, 109-119, https://doi.org/10.1016/j.quaint.2016.10.039, 2018.

Benson, L., Kashgarian, M., Rye, R., Lund, S., Paillet, F., Smoot, J., Kester, C., Mensing, S., Meko, D., and Lindström, S.: Holocene 
multidecadal and multicentennial droughts affecting Northern California and Nevada, Quaternary Sci. Rev., 21, 659-682, https://doi.org/10.1016/S0277-3791(01)00048-8, 2002.

Berger, A. and Loutre, M. F.: Insolation values for the climate of the last 10 million years, Quaternary Sci. Rev., 10, 297-317, https://doi.org/10.1016/0277-3791(91)90033-Q, 1991.

Bernal, J. P., Lachniet, M., McCulloch, M., Mortimer, G., Morales, P., and Cienfuegos, E.: A speleothem record of Holocene climate variability from southwestern Mexico, Quaternary Res., 75, 104113, https://doi.org/10.1016/j.yqres.2010.09.002, 2011.

Bhattacharya, T., Byrne, R., Böhnel, H., Wogau, K., Kienel, U., Ingram, B. L., and Zimmerman, S.: Cultural implications of late Holocene climate change in the Cuenca Oriental, Mexico, P. Natl. Acad. Sci. USA, 112, 1693-1698, https://doi.org/10.1073/pnas.1405653112, 2015.

Bhattacharya, T., Tierney, J. E., Addison, J. A., and Murray, J. W.: Ice-sheet modulation of deglacial North American monsoon intensification, Nat. Geosci., 11, 848-852, https://doi.org/10.1038/s41561-018-0220-7, 2018.

Blaauw, M., Christen, J. A., Bennett, K. D., and Reimer, P. J.: Double the dates and go for Bayes - Impacts of model choice, dating density and quality on chronologies, Quaternary Sci. Rev., 188, 58-66, https://doi.org/10.1016/j.quascirev.2018.03.032, 2018.

Boldt, B. R., Kaufman, D. S., McKay, N. P., and Briner, J. P.: Holocene summer temperature reconstruction from sedimentary chlorophyll content, with treatment of age uncertainties, Kurupa Lake, Arctic Alaska, Holocene, 25, 641-650, https://doi.org/10.1177/0959683614565929, 2015.

Boos, D. D.: Introduction to the bootstrap world, Statist. Sci., 18, 168-174, https://doi.org/10.1214/ss/1063994971, 2003.

Bradley, R. S.: Paleoclimatology: reconstructing climates of the Quaternary, Elsevier, San Diego, CA, USA, 2015.

Bringué, M. and Rochon, A.: Late Holocene paleoceanography and climate variability over the Mackenzie Slope (Beaufort Sea, Canadian Arctic), Mar. Geol., 291-294, 83-96, https://doi.org/10.1016/j.margeo.2011.11.004, 2012.

Brown, K. J. and Hebda, R. J.: Origin, development, and dynamics of coastal temperate conifer rainforests of southern Vancouver Island, Canada, Can. J. Forest Res., 32, 353-372, https://doi.org/10.1139/x01-197, 2002.

Brown, K. J. and Schoups, G.: Multi-millennial streamflow dynamics in two forested watersheds on Vancouver Island, Canada, Quaternary Res., 83, 415-426, https://doi.org/10.1016/j.yqres.2015.03.003, 2015.

Brown, K. J., Fitton, R. J., Schoups, G., Allen, G. B., Wahl, K. A., and Hebda, R. J.: Holocene precipitation in the coastal temperate rainforest complex of southern British Columbia, Canada, Quaternary Sci. Rev., 25, 2762-2779, https://doi.org/10.1016/j.quascirev.2006.02.020, 2006.

Brown, K. J., Hebda, N., Schoups, G., Conder, N., Smith, K., and Trofymow, J.: Long-term climate, vegetation and fire regime change in a managed municipal water supply area, British Columbia, Canada, Holocene, 29, 1411-1424, https://doi.org/10.1177/0959683619854523, 2019.

Brubaker, L. B., Garfinkel, H. L., and Edwards, M. E.: A Late Wisconsin and Holocene vegetation history from the Central Brooks Range: Implications for Alaskan palaeoecology, Quaternary Res., 20, 194-214, https://doi.org/10.1016/00335894(83)90077-7, 1983.
Bunbury, J. and Gajewski, K.: Postglacial climates inferred from a lake at treeline, southwest Yukon Territory, Canada, Quaternary Sci. Rev., 28, 354-369, https://doi.org/10.1016/j.quascirev.2008.10.007, 2009.

Chakraborty, K., Finkelstein, S. A., Desloges, J. R., and Chow, N. A.: Holocene paleoenvironmental changes inferred from diatom assemblages in sediments of Kusawa Lake, Yukon Territory, Canada, Quaternary Res., 74, 15-22, https://doi.org/10.1016/j.yqres.2010.04.011, 2010.

Chase, M., Bleskie, C., Walker, I. R., Gavin, D. G., and Hu, F. S.: Midge-inferred Holocene summer temperatures in Southeastern British Columbia, Canada, Palaeogeogr. Palaeocl., 257, 244259, https://doi.org/10.1016/j.palaeo.2007.10.020, 2008.

Clegg, B. F. and Hu, F. S.: An oxygen-isotope record of Holocene climate change in the south-central Brooks Range, Alaska, Quaternary Sci. Rev., 29, 928-939, https://doi.org/10.1016/j.quascirev.2009.12.009, 2010.

Clegg, B. F., Clarke, G. H., Chipman, M. L., Chou, M., Walker, I. R., Tinner, W., and Hu, F. S.: Six millennia of summer temperature variation based on midge analysis of lake sediments from Alaska, Quaternary Sci. Rev., 29, 3308-3316, https://doi.org/10.1016/j.quascirev.2010.08.001, 2010.

Clegg, B. F., Kelly, R., Clarke, G. H., Walker, I. R., and Hu, F. S.: Nonlinear response of summer temperature to Holocene insolation forcing in Alaska, P. Natl. Acad. Sci. USA, 108, 1929919304, https://doi.org/10.1073/pnas.1110913108, 2011.

Cole, K. L. and Liu, G.-W.: Holocene paleoecology of an estuary on Santa Rosa Island, California, Quaternary Res., 41, 326-335, https://doi.org/10.1006/qres.1994.1037, 1994.

Cumming, B. F., Laird, K. R., Bennett, J. R., Smol, J. P., and Salomon, A. K.: Persistent millennial-scale shifts in moisture regimes in western Canada during the past six millennia, P. Natl. Acad. Sci. USA, 99, 16117-16121, https://doi.org/10.1073/pnas.252603099, 2002.

Cwynar, L. C.: A Late-Quaternary vegetation history from Hanging Lake, Northern Yukon, Ecol. Monogr., 52, 1-24, https://doi.org/10.2307/2937342, 1982.

Cwynar, L. C.: A late Quaternary vegetation history from Lily Lake, Chilkat Peninsula, southeast Alaska, Can. J. Botany, 68, 11061112, https://doi.org/10.1139/b90-139, 1990.

Cwynar, L. C. and Spear, R. W.: Reversion of forest to tundra in the Central Yukon, Ecology, 72, 202-212, https://doi.org/10.2307/1938915, 1991.

Cwynar, L. C. and Spear, R. W.: Paleovegetation and paleoclimatic changes in the Yukon at 6ka BP, Géogr. Phys. Quatern., 49, 2935, https://doi.org/10.7202/033027ar, 2007.

de Vernal, A., Hillaire-Marcel, C., and Darby, D. A.: Variability of sea ice cover in the Chukchi Sea (western Arctic Ocean) during the Holocene, Paleoceanography, 20, PA4018, https://doi.org/10.1029/2005PA001157, 2005.

de Vernal, A., Hillaire-Marcel, C., Rochon, A., Fréchette, B., Henry, M., Solignac, S., and Bonnet, S.: Dinocyst-based reconstructions of sea ice cover concentration during the Holocene in the Arctic Ocean, the northern North Atlantic Ocean and its adjacent seas, Quaternary Sci. Rev., 79, 111-121, https://doi.org/10.1016/j.quascirev.2013.07.006, 2013.

Du, X., Hendy, I., and Schimmelmann, A.: A 9000-year flood history for Southern California: A revised stratigraphy of varved 
sediments in Santa Barbara Basin, Mar. Geol., 397, 29-42, https://doi.org/10.1016/j.margeo.2017.11.014, 2018.

Ersek, V., Clark, P. U., Mix, A. C., Cheng, H., and Lawrence Edwards, R.: Holocene winter climate variability in midlatitude western North America, Nat. Commun., 3, 1219, https://doi.org/10.1038/ncomms2222, 2012.

Fall, P. L.: Holocene dynamics of the subalpine forest in central Colorado, American Association of Stratigraphic Palynologists Contribution Series, 16, 31-46, 1985.

Fall, P. L.: Vegetation dynamics in the southern Rocky Mountains: Late Pleistocene and Holocene timberline fluctuations, $\mathrm{PhD}$ thesis, University of Arizona, Tucson, USA, p. 303, 1988.

Fall, P. L.: Timberline fluctuations and late Quaternary paleoclimates in the Southern Rocky Mountains, Colorado, Geol. Soc. Am. Bull., 109, 1306-1320, https://doi.org/10.1130/00167606(1997)109<1306:TFALQP>2.3.CO;2, 1997.

Farmer, J. R., Cronin, T. M., de Vernal, A., Dwyer, G. S., Keigwin, L. D., and Thunell, R. C.: Western Arctic Ocean temperature variability during the last 8000 years, Geophys. Res. Lett., 38, L24602, https://doi.org/10.1029/2011GL049714, 2011.

Finkenbinder, M. S., Abbott, M. B., Edwards, M. E., Langdon, C. T., Steinman, B. A., and Finney, B. P.: A 31,000 year record of paleoenvironmental and lake-level change from Harding Lake, Alaska, USA, Quaternary Sci. Rev., 87, 98-113, https://doi.org/10.1016/j.quascirev.2014.01.005, 2014.

Finney, B. P., Bigelow, N. H., Barber, V. A., and Edwards, M. E.: Holocene climate change and carbon cycling in a groundwaterfed, boreal forest lake: Dune Lake, Alaska, J. Paleolimnol., 48, 43-54, https://doi.org/10.1007/s10933-012-9617-2, 2012.

Fisher, D., Osterberg, E., Dyke, A., Dahl-Jensen, D., Demuth, M., Zdanowicz, C., Bourgeois, J., Koerner, R. M., Mayewski, P., Wake, C., Kreutz, K., Steig, E., Zheng, J., Yalcin, K., Goto-Azuma, K., Luckman, B., and Rupper, S.: The Mt Logan Holocene - late Wisconsinan isotope record: tropical Pacific-Yukon connections, Holocene, 18, 667-677, https://doi.org/10.1177/0959683608092236, 2008.

Flower, B. P., Hastings, D. W., Hill, H. W., and Quinn, T. M.: Phasing of deglacial warming and Laurentide Ice Sheet meltwater in the Gulf of Mexico, Geology, 32, 597, https://doi.org/10.1130/G20604.1, 2004.

Gajewski, K., Mott, R. J., Ritchie, J. C., and Hadden, K.: Holocene vegetation history of Banks Island, Northwest Territories, Canada, Can. J. Botany, 78, 430-436, https://doi.org/10.1139/b00-018, 2000.

Galloway, J. M., Lenny, A. M., and Cumming, B. F.: Hydrological change in the central interior of British Columbia, Canada: diatom and pollen evidence of millennial-to-centennial scale change over the Holocene, J. Paleolimnol., 45, 183-197, https://doi.org/10.1007/s10933-010-9490-9, 2011.

Garfin, A.: Assessment of climate change in the southwest United States: a report prepared for the National Climate Assessment, Island Press, Washington DC, 2013.

Gavin, D. G., Henderson, A. C. G., Westover, K. S., Fritz, S. C., Walker, I. R., Leng, M. J., and Hu, F. S.: Abrupt Holocene climate change and potential response to solar forcing in western Canada, Quaternary Sci. Rev., 30, 1243-1255, https://doi.org/10.1016/j.quascirev.2011.03.003, 2011.

Goman, M., Joyce, A., Lund, S., Pearson, C., Guerra, W., Dale, D., Hammond, D. E., and Celestian, A. J.: Preliminary results from Laguna Minucúa: a potentially annually resolved record of climate and environmental change for the past $\sim 5000$ years in the Mixteca Alta of Oaxaca, Mexico, Quatern. Int., 469, 85-95, https://doi.org/10.1016/j.quaint.2017.01.027, 2018.

Guiot, J. and de Vernal, A.: Chapter Thirteen. Transfer functions: Methods for quantitative paleoceanography based on microfossils, in: Developments in Marine Geology, edited by: HillaireMarcel, C. and De Vernal, A., Elsevier, Amsterdam, Netherlands, 523-563, 2007.

Harbert, R. S. and Nixon, K. C.: Quantitative Late Quaternary climate reconstruction from plant macrofossil communities in western North America, Open Quaternary, 4, 8, https://doi.org/10.5334/oq.46, 2018.

Heiser, C., McKay, N. P., Simpson, G. A., and Routson, C. C.: nickmckay/LiPD-utilities: v0.2.5.5, Zenodo, https://doi.org/10.5281/zenodo.1256889, 2018.

Hill, T. M., Kennett, J. P., Pak, D. K., Behl, R. J., Robert, C., and Beaufort, L.: Pre-Bølling warming in Santa Barbara Basin, California: surface and intermediate water records of early deglacial warmth, Quaternary Sci. Rev., 25, 2835-2845, https://doi.org/10.1016/j.quascirev.2006.03.012, 2006.

Hodell, D. A., Curtis, J. H., and Brenner, M.: Possible role of climate in the collapse of Classic Maya civilization, Nature, 375, 391-394, https://doi.org/10.1038/375391a0, 1995.

Hu, F. S., Ito, E., Brubaker, L. B., and Anderson, P. M.: Ostracode geochemical record of Holocene climatic change and implications for vegetational response in the Northwestern Alaska Range, Quaternary Res., 49, 86-95, https://doi.org/10.1006/qres.1997.1936, 1998.

Hughes, M. K. and Graumlich, L. J.: Multi-millennial dendroclimatic studies from the western United States, in: Climatic variations and forcing mechanisms of the last 2000 years, Springer, Berlin, Heidelberg, 109-124, 1996.

Irvine, F., Cwynar, L. C., Vermaire, J. C., and Rees, A. B. H.: Midge-inferred temperature reconstructions and vegetation change over the last 15,000 years from Trout Lake, northern Yukon Territory, eastern Beringia, J. Paleolimnol., 48, 133-146, https://doi.org/10.1007/s10933-012-9612-7, 2012.

Jiménez-Moreno, G. and Anderson, R. S.: Pollen and macrofossil evidence of Late Pleistocene and Holocene treeline fluctuations from an alpine lake in Colorado, USA, Holocene, 23, 68-77, https://doi.org/10.1177/0959683612450199, 2013.

Jiménez-Moreno, G., Fawcett, P. J., and Scott Anderson, R.: Millennial- and centennial-scale vegetation and climate changes during the late Pleistocene and Holocene from northern New Mexico (USA), Quaternary Sci. Rev., 27, 1442-1452, https://doi.org/10.1016/j.quascirev.2008.04.004, 2008.

Jimenez-Moreno, G., Anderson, R. S., Atudorei, V., and Toney, J. L.: A high-resolution record of climate, vegetation, and fire in the mixed conifer forest of northern Colorado, USA, Geol. Soc. Am. Bull., 123, 240-254, https://doi.org/10.1130/B30240.1, 2011.

Jiménez-Moreno, G., Anderson, R. S., Shuman, B. N., and Yackulic, E.: Forest and lake dynamics in response to temperature, North American monsoon and ENSO variability during the Holocene in Colorado (USA), Quaternary Sci. Rev., 211, 59-72, https://doi.org/10.1016/j.quascirev.2019.03.013, 2019.

Johnson, B. G., Jiménez-Moreno, G., Eppes, M. C., Diemer, J. A., and Stone, J. R.: A multiproxy record of postglacial climate variability from a shallowing, 12-m deep sub-alpine bog in the south- 
eastern San Juan Mountains of Colorado, USA, Holocene, 23, 1028-1038, https://doi.org/10.1177/0959683613479682, 2013.

Jones, M. D., Metcalfe, S. E., Davies, S. J., and Noren, A.: Late Holocene climate reorganisation and the North American Monsoon, Quaternary Sci. Rev., 124, 290-295, https://doi.org/10.1016/j.quascirev.2015.07.004, 2015.

Juggins, S. and Birks, H. J. B.: Quantitative environmental reconstructions from biological data, in: Tracking Environmental Change Using Lake Sediments: Data Handling and Numerical Techniques, edited by: Birks, H. J. B., Lotter, A. F., Juggins, S., and Smol, J. P., Springer, Dordrecht, The Netherlands, 431-494, 2012.

Kaufman, D., Axford, Y., Anderson, R. S., Lamoureux, S. F., Schindler, D. E., Walker, I. R., and Werner, A.: A multi-proxy record of the Last Glacial Maximum and last 14,500 years of paleoenvironmental change at Lone Spruce Pond, southwestern Alaska, J. Paleolimnol., 48, 9-26, https://doi.org/10.1007/s10933-012-9607-4, 2012.

Kaufman, D., McKay, N., Routson, C., Erb, M., Davis, B., Heiri, O., Jaccard, S., Tierney, J., Dätwyler, C., Axford, Y., Brussel, T., Cartapanis, O., Chase, B., Dawson, A., de Vernal, A., Engels, S., Jonkers, L., Marsicek, J., Moffa-Sánchez, P., Morrill, C., Orsi, A., Rehfeld, K., Saunders, K., Sommer, P. S., Thomas, E., Tonello, M., Tóth, M., Vachula, R., Andreev, A., Bertrand, S., Biskaborn, B., Bringué, M., Brooks, S., Caniupán, M., Chevalier, M., Cwynar, L., Emile-Geay, J., Fegyveresi, J., Feurdean, A., Finsinger, W., Fortin, M.-C., Foster, L., Fox, M., Gajewski, K., Grosjean, M., Hausmann, S., Heinrichs, M., Holmes, N., Ilyashuk, B., Ilyashuk, E., Juggins, S., Khider, D., Koinig, K., Langdon, P., Larocque-Tobler, I., Li, J., Lotter, A., Luoto, T., Mackay, A., Magyari, E., Malevich, S., Mark, B., Massaferro, J., Montade, V., Nazarova, L., Novenko, E., Pařil, P., Pearson, E., Peros, M., Pienitz, R., Płóciennik, M., Porinchu, D., Potito, A., Rees, A., Reinemann, S., Roberts, S., Rolland, N., Salonen, S., Self, A., Seppä, H., Shala, S., St-Jacques, J.-M., Stenni, B., Syrykh, L., Tarrats, P., Taylor, K., van den Bos, V., Velle, G., Wahl, E., Walker, I., Wilmshurst, J., Zhang, E., and Zhilich, S.: A global database of Holocene paleotemperature records, Sci. Data, 7, 115, https://doi.org/10.1038/s41597-020-0445-3, 2020a.

Kaufman, D., McKay, N., Routson, C., Erb, M., Dätwyler, C., Sommer, P. S., Heiri, O., and Davis, B.: Holocene global mean surface temperature, a multi-method reconstruction approach, Sci. Data, 7, 201, https://doi.org/10.1038/s41597-020-0530-7, 2020b.

Kennett, D. J., Kennett, J. P., Erlandson, J. M., and Cannariato, K. G.: Human responses to Middle Holocene climate change on California's Channel Islands, Quaternary Sci. Rev., 26, 351-367, https://doi.org/10.1016/j.quascirev.2006.07.019, 2007.

Kirby, M. E., Zimmerman, S. R. H., Patterson, W. P., and Rivera, J. J.: A 9170-year record of decadal-to-multi-centennial scale pluvial episodes from the coastal southwest United States: a role for atmospheric rivers?, Quaternary Sci. Rev., 46, 57-65, https://doi.org/10.1016/j.quascirev.2012.05.008, 2012.

Kirby, M. E., Knell, E. J., Anderson, W. T., Lachniet, M. S., Palermo, J., Eeg, H., Lucero, R., Murrieta, R., Arevalo, A., Silveira, E., and Hiner, C. A.: Evidence for insolation and Pacific forcing of late glacial through Holocene climate in the Central Mojave Desert (Silver Lake, CA), Quaternary Res., 84, 174-186, https://doi.org/10.1016/j.yqres.2015.07.003, 2015.
Kirby, M. E. C., Patterson, W. P., Lachniet, M., Noblet, J. A., Anderson, M. A., Nichols, K., and Avila, J.: Pacific southwest United States Holocene droughts and pluvials inferred from sediment $\delta 18 \mathrm{O}$ (calcite) and grain size data (Lake Elsinore, California), Front. Earth Sci., 7, 74, https://doi.org/10.3389/feart.2019.00074, 2019.

Konecky, B. L., McKay, N. P., Churakova (Sidorova), O. V., ComasBru, L., Dassié, E. P., DeLong, K. L., Falster, G. M., Fischer, M. J., Jones, M. D., Jonkers, L., Kaufman, D. S., Leduc, G., Managave, S. R., Martrat, B., Opel, T., Orsi, A. J., Partin, J. W., Sayani, H. R., Thomas, E. K., Thompson, D. M., Tyler, J. J., Abram, N. J., Atwood, A. R., Cartapanis, O., Conroy, J. L., Curran, M. A., Dee, S. G., Deininger, M., Divine, D. V., Kern, Z., Porter, T. J., Stevenson, S. L., von Gunten, L., and Iso2k Project Members: The Iso2k database: a global compilation of paleo- $\delta^{18} \mathrm{O}$ and $\delta^{2} \mathrm{H}$ records to aid understanding of Common Era climate, Earth Syst. Sci. Data, 12, 2261-2288, https://doi.org/10.5194/essd-12-22612020, 2020.

Lachniet, M. S., Denniston, R. F., Asmerom, Y., and Polyak, V. J.: Orbital control of western North America atmospheric circulation and climate over two glacial cycles, Nat. Commun., 5, 3805, https://doi.org/10.1038/ncomms4805, 2014.

Larsen, D. J., Finkenbinder, M. S., Abbott, M. B., and Ofstun, A. R.: Deglaciation and postglacial environmental changes in the Teton Mountain Range recorded at Jenny Lake, Grand Teton National Park, WY, Quaternary Sci. Rev., 138, 62-75, https://doi.org/10.1016/j.quascirev.2016.02.024, 2016.

Lemmen, J. and Lacourse, T.: Fossil chironomid assemblages and inferred summer temperatures for the past 14,000 years from a low-elevation lake in Pacific Canada, J. Paleolimnol., 59, 427442, https://doi.org/10.1007/s10933-017-9998-3, 2018.

Levy, L. B., Kaufman, D. S., and Werner, A.: Holocene glacier fluctuations, Waskey Lake, northeastern Ahklun Mountains, southwestern Alaska, Holocene, 14, 185-193, https://doi.org/10.1191/0959683604hl675rp, 2004.

Lopes, C. and Mix, A. C.: North Pacific paleotemperature and paleoproductivity reconstructions based on diatom species, Paleoceanography and Paleoclimatology, 33, 703-715, https://doi.org/10.1029/2018PA003352, 2018.

Lundeen, Z., Brunelle, A., Burns, S. J., Polyak, V., and Asmerom, Y.: A speleothem record of Holocene paleoclimate from the northern Wasatch Mountains, southeast Idaho, USA, Quatern. Int., 310, 83-95, https://doi.org/10.1016/j.quaint.2013.03.018, 2013.

Lynch, E. A.: Origin of a park-forest vegetation mosaic in the Wind River Range, Wyoming, Ecology, 79, 1320-1338, https://doi.org/10.1890/00129658(1998)079[1320:OOAPFV]2.0.CO;2, 1998.

MacDonald, G. M.: Postglacial vegetation history of the Mackenzie River Basin, Quaternary Res., 28, 245-262, https://doi.org/10.1016/0033-5894(87)90063-9, 1987.

MacDonald, G. M. and Cwynar, L. C.: A fossil pollen based reconstruction of the late Quaternary history of lodgepole pine (Pinus contorta ssp. latifolia) in the western interior of Canada, Can. J. Forest Res., 15, 1039-1044, https://doi.org/10.1139/x85-168, 1985.

MacDonald, G. M., Moser, K. A., Bloom, A. M., Potito, A. P., Porinchu, D. F., Holmquist, J. R., Hughes, J., and Kremenetski, K. V.: Prolonged California aridity linked to climate warm- 
ing and Pacific sea surface temperature, Sci. Rep., 6, 33325, https://doi.org/10.1038/srep33325, 2016.

Maher, L. J.: Pollen analyses of surface materials from the southern San Juan Mountains, Colorado, Geol. Soc. Am. Bull., 74, 1485, https://doi.org/10.1130/00167606(1963)74[1485:PAOSMF]2.0.CO;2, 1963.

Maher, L. J.: Absolute pollen diagram of Redrock Lake, Boulder County, Colorado, Quaternary Res., 2, 531-553, https://doi.org/10.1016/0033-5894(72)90090-7, 1972.

Mann, D. H., Heiser, P. A., and Finney, B. P.: Holocene history of the Great Kobuk Sand Dunes, Northwestern Alaska, Quaternary Sci. Rev., 21, 709-731, https://doi.org/10.1016/S02773791(01)00120-2, 2002.

Mantua, N. J., Hare, S. R., Zhang, Y., Wallace, J. M., and Francis, R. C.: A Pacific interdecadal climate oscillation with impacts on salmon production, B. Am. Meteorol. Soc., 78, 1069-1079, https://doi.org/10.1175/15200477(1997)078<1069:APICOW>2.0.CO;2, 1997.

Marchitto, T. M., Muscheler, R., Ortiz, J. D., Carriquiry, J. D., and van Geen, A.: Dynamical response of the Tropical Pacific Ocean to solar forcing during the early Holocene, Science, 330, 13781381, https://doi.org/10.1126/science.1194887, 2010.

Marcott, S. A., Shakun, J. D., Clark, P. U., and Mix, A. C.: A reconstruction of regional and global temperature for the past 11,300 years, Science, 339, 1198-1201, https://doi.org/10.1126/science.1228026, 2013.

Marlon, J. R., Bartlein, P. J., Long, C., Gavin, D. G., Anderson, R. S., and Briles, C.: Natural versus human causes of fire in the western US, Proc. Natl. Acad. Sci. USA, 109, 535-543, 2012.

Marsicek, J., Shuman, B., Brewer, S., Foster, D. R., and Oswald, W. W.: Moisture and temperature changes associated with the mid-Holocene Tsuga decline in the northeastern United States, Quaternary Sci. Rev., 80, 129-142, https://doi.org/10.1016/j.quascirev.2013.09.001, 2013.

Marsicek, J., Shuman, B. N., Bartlein, P. J., Shafer, S. L., and Brewer, S.: Reconciling divergent trends and millennial variations in Holocene temperatures, Nature, 554, 92-96, https://doi.org/10.1038/nature25464, 2018.

Mathewes, R. W.: A palynological study of postglacial vegetation changes in the University Research Forest, southwestern British Columbia, Can. J. Botany, 51, 2085-2103, https://doi.org/10.1139/b73-271, 1973.

McAfee, S. A. and Russell, J. L.: Northern Annular Mode impact on spring climate in the western United States, Geophys. Res. Lett., 35, L17701. https://doi.org/10.1029/2008GL034828, 2008.

McClymont, E. L., Ganeshram, R. S., Pichevin, L. E., Talbot, H. M., van Dongen, B. E., Thunell, R. C., Haywood, A. M., Singarayer, J. S., and Valdes, P. J.: Sea-surface temperature records of Termination 1 in the Gulf of California: Challenges for seasonal and interannual analogues of tropical Pacific climate change, Paleoceanography, 27, PA2202, https://doi.org/10.1029/2011PA002226, 2012.

McGann, M.: High-resolution foraminiferal, isotopic, and trace element records from Holocene estuarine deposits of San Francisco Bay, California, J. Coastal Res., 245, 1092-1109, https://doi.org/10.2112/08A-0003.1, 2008.

McKay, N. P. and Emile-Geay, J.: Technical note: The Linked Paleo Data framework - a common tongue for paleoclimatology,
Clim. Past, 12, 1093-1100, https://doi.org/10.5194/cp-12-10932016, 2016.

McKay, N. P. and Kaufman, D. S.: Holocene climate and glacier variability at Hallet and Greyling Lakes, Chugach Mountains, south-central Alaska, J. Paleolimnol., 41, 143-159, https://doi.org/10.1007/s10933-008-9260-0, 2009.

Metcalfe, S. E., Barron, J. A., and Davies, S. J.: The Holocene history of the North American Monsoon: "known knowns" and "known unknowns" in understanding its spatial and temporal complexity, Quaternary. Sci. Rev., 120, 1-27, https://doi.org/10.1016/j.quascirev.2015.04.004, 2015.

Michels, A., Laird, K. R., Wilson, S. E., Thomson, D., Leavitt, P. R., Oglesby, R. J., and Cumming, B. F.: Multidecadal to millennial-scale shifts in drought conditions on the Canadian prairies over the past six millennia: implications for future drought assessment, Glob. Change Biol., 13, 1295-1307, https://doi.org/10.1111/j.1365-2486.2007.01367.x, 2007.

Minckley, T. A., Shriver, R. K., and Shuman, B.: Resilience and regime change in a southern Rocky Mountain ecosystem during the past 17000 years, Ecol. Monogr., 82, 49-68, https://doi.org/10.1890/11-0283.1, 2012.

Morris, J. L., Brunelle, A., DeRose, R. J., Seppä, H., Power, M. J., Carter, V., and Bares, R.: Using fire regimes to delineate zones in a high-resolution lake sediment record from the western United States, Quaternary Res., 79, 24-36, https://doi.org/10.1016/j.yqres.2012.10.002, 2013.

Muhs, D. R., Budahn, J. R., McGeehin, J. P., Bettis, E. A., Skipp, G., Paces, J. B., and Wheeler, E. A.: Loess origin, transport, and deposition over the past 10,000 years, WrangellSt. Elias National Park, Alaska, Aeolian Res., 11, 85-99, https://doi.org/10.1016/j.aeolia.2013.06.001, 2013.

Munroe, J. S., McElroy, R., O’Keefe, S., Peters, A., and Wasson, L.: Holocene records of eolian dust deposition from high-elevation lakes in the Uinta Mountains, Utah, USA, J. Quaternary Sci., 36, 66-75, https://doi.org/10.1002/jqs.3250, 2020.

Nelson, D. B., Abbott, M. B., Steinman, B., Polissar, P. J., Stansell, N. D., Ortiz, J. D., Rosenmeier, M. F., Finney, B. P., and Riedel, J.: Drought variability in the Pacific Northwest from a 6,000-yr lake sediment record, P. Natl. Acad. Sci. USA, 108, 3870-3875, https://doi.org/10.1073/pnas.1009194108, 2011.

Nichols, J. E., Peteet, D. M., Moy, C. M., Castañeda, I. S., McGeachy, A., and Perez, M.: Impacts of climate and vegetation change on carbon accumulation in a south-central Alaskan peatland assessed with novel organic geochemical techniques, Holocene, 24, 1146-1155, https://doi.org/10.1177/0959683614540729, 2014.

Ohlwein, C. and Wahl, E. R.: Review of probabilistic pollenclimate transfer methods, Quaternary Sci. Rev., 31, 17-29, https://doi.org/10.1016/j.quascirev.2011.11.002, 2012.

PAGES 2k Consortium: A global multiproxy database for temperature reconstructions of the Common Era, Sci. Data, 4, 170088, https://doi.org/10.1038/sdata.2017.88, 2017.

Palmer, S., Walker, I., Heinrichs, M., Hebda, R., and Scudder, G.: Postglacial midge community change and Holocene palaeotemperature reconstructions near treeline, southern British Columbia (Canada), J. Paleolimnol., 28, 469-490, https://doi.org/10.1023/A:1021644122727, 2002.

Pellatt, M. G. and Mathewes, R. W.: Paleoecology of postglacial tree line fluctuations on the Queen 
Charlotte Islands, Canada, Écoscience, 1, 71-81, https://doi.org/10.1080/11956860.1994.11682230, 1994.

Pellatt, M. G., Smith, M. J., Mathewes, R. W., Walker, I. R., and Palmer, S. L.: Holocene treeline and climate change in the subalpine zone near Stoyoma Mountain, Cascade Mountains, southwestern British Columbia, Canada, Arct. Antarct. Alp. Res., 32, 73-83, https://doi.org/10.1080/15230430.2000.12003341, 2000.

Petersen, K. L.: Palynology in Montezuma County, southwestern Colorado: The local history of pinyon pine (Pinus edulis), ASSP Contribution Series, 16, 47-62, 1985.

Pompeani, D. P., Steinman, B. A., and Abbott, M. B.: A sedimentary and geochemical record of water-level changes from Rantin Lake, Yukon, Canada, J. Paleolimnol., 48, 147-158, https://doi.org/10.1007/s10933-012-9602-9, 2012.

Poore, R. Z., Dowsett, H. J., Verardo, S., and Quinn, T. M.: Millennial- to century-scale variability in Gulf of Mexico Holocene climate records, Paleoceanography, 18, 1048, https://doi.org/10.1029/2002PA000868, 2003.

Poore, R. Z., Pavich, M. J., and Grissino-Mayer, H. D.: Record of the North American southwest monsoon from Gulf of Mexico sediment cores, Geology, 33, 209, https://doi.org/10.1130/G21040.1, 2005.

Porter, T. J., Schoenemann, S. W., Davies, L. J., Steig, E. J., Bandara, S., and Froese, D. G.: Recent summer warming in northwestern Canada exceeds the Holocene thermal maximum, Nat. Commun., 10, 1631, https://doi.org/10.1038/s41467-019-09622y, 2019.

Potito, A. P., Porinchu, D. F., MacDonald, G. M., and Moser, K. A.: A late Quaternary chironomid-inferred temperature record from the Sierra Nevada, California, with connections to northeast Pacific sea surface temperatures, Quaternary Res., 66, 356-363, https://doi.org/10.1016/j.yqres.2006.05.005, 2006.

Power, M. J., Marlon, J., Ortiz, N., Bartlein, P. J., Harrison, S. P., Mayle, F. E., Ballouche, A., Bradshaw, R. H. W., Carcaillet, C., Cordova, C., Mooney, S., Moreno, P. I., Prentice, I. C., Thonicke, K., Tinner, W., Whitlock, C., Zhang, Y., Zhao, Y., Ali, A. A., Anderson, R. S., Beer, R., Behling, H., Briles, C., Brown, K. J., Brunelle, A., Bush, M., Camill, P., Chu, G. Q., Clark, J., Colombaroli, D., Connor, S., Daniau, A.-L., Daniels, M., Dodson, J., Doughty, E., Edwards, M. E., Finsinger, W., Foster, D., Frechette, J., Gaillard, M.-J., Gavin, D. G., Gobet, E., Haberle, S., Hallett, D. J., Higuera, P., Hope, G., Horn, S., Inoue, J., Kaltenrieder, P., Kennedy, L., Kong, Z. C., Larsen, C., Long, C. J., Lynch, J., Lynch, E. A., McGlone, M., Meeks, S., Mensing, S., Meyer, G., Minckley, T., Mohr, J., Nelson, D. M., New, J., Newnham, R., Noti, R., Oswald, W., Pierce, J., Richard, P. J. H., Rowe, C., Sanchez Goñi, M. F., Shuman, B. N., Takahara, H., Toney, J., Turney, C., Urrego-Sanchez, D. H., Umbanhowar, C., Vandergoes, M., Vanniere, B., Vescovi, E., Walsh, M., Wang, X., Williams, N., Wilmshurst, J., and Zhang, J. H.: Changes in fire regimes since the Last Glacial Maximum: an assessment based on a global synthesis and analysis of charcoal data, Clim. Dynam., 30, 887-907, https://doi.org/10.1007/s00382-007-0334-x, 2008.

Praetorius, S. K., Mix, A. C., Walczak, M. H., Wolhowe, M. D., Addison, J. A., and Prahl, F. G.: North Pacific deglacial hypoxic events linked to abrupt ocean warming, Nature, 527, 362-366, https://doi.org/10.1038/nature15753, 2015.
Praetorius, S. K., Condron, A., Mix, A. C., Walczak, M. H., McKay, J. L., and Du, J.: The role of Northeast Pacific meltwater events in deglacial climate change, Sci. Adv., 6, eaay2915, https://doi.org/10.1126/sciadv.aay2915, 2020.

Pribyl, P. and Shuman, B. N.: A computational approach to Quaternary lake-level reconstruction applied in the central Rocky Mountains, Wyoming, USA, Quaternary Res., 82, 249-259, https://doi.org/10.1016/j.yqres.2014.01.012, 2014.

Rainville, R. A. and Gajewski, K.: Holocene environmental history of the Aishihik Region, Yukon, Canada, Can. J. Earth Sci., 50, 397-405, https://doi.org/10.1139/cjes-2012-0103, 2013.

Redmond, K. T. and Koch, R. W.: Surface climate and streamflow variability in the Western United States and their relationship to large-scale circulation indices, Water Resour. Res., 27, 23812399, https://doi.org/10.1029/91WR00690, 1991.

Reinemann, S. A., Porinchu, D. F., Bloom, A. M., Mark, B. G., and Box, J. E.: A multi-proxy paleolimnological reconstruction of Holocene climate conditions in the Great Basin, United States, Quaternary Res., 72, 347-358, https://doi.org/10.1016/j.yqres.2009.06.003, 2009.

Ritchie, J. C.: The Modern and Late Quaternary vegetation of the Campbell-Dolomite Uplands, near Inuvik, N.W.T., Canada, Ecol. Monogr., 47, 401-423, https://doi.org/10.2307/1942175, 1977.

Rodysill, J. R., Anderson, L., Cronin, T. M., Jones, M. C., Thompson, R. S., Wahl, D. B., Willard, D. A., Addison, J. A., Alder, J. R., Anderson, K. H., Anderson, L., Barron, J. A., Bernhardt, C. E., Hostetler, S. W., Kehrwald, N. M., Khan, N. S., Richey, J. N., Starratt, S. W., Strickland, L. E., Toomey, M. R., Treat, C. C., and Wingard, G. L.: A North American Hydroclimate Synthesis (NAHS) of the Common Era, Glob. Planet. Change, 162, 175-198, https://doi.org/10.1016/j.gloplacha.2017.12.025, 2018.

Rosenberg, S. M., Walker, I. R., Mathewes, R. W., and Hallett, D. J.: Midge-inferred Holocene climate history of two subalpine lakes in southern British Columbia, Canada, Holocene, 14, 258-271, https://doi.org/10.1191/0959683604hl703rp, 2004.

Routson, C. C. and McKay, N. P.: A multiproxy database of western North American Holocene paleoclimate records, figshare dataset, https://doi.org/10.6084/m9.figshare.12863843.v1, 2020.

Routson, C. C., McKay, N. P., Kaufman, D. S., Erb, M. P., Goosse, H., Shuman, B. N., Rodysill, J. R., and Ault, T.: Mid-latitude net precipitation decreased with Arctic warming during the Holocene, Nature, 568, 83-87, https://doi.org/10.1038/s41586019-1060-3, 2019a.

Routson, C. C., Arcusa, S. H., McKay, N. P., and Overpeck, J. T.: A 4,500-year-long record of southern Rocky Mountain dust deposition, Geophys. Res. Lett., 46, 8281-8288, https://doi.org/10.1029/2019GL083255, 2019b.

Salzer, M. W., Bunn, A. G., Graham, N. E., and Hughes, M. K.: Five millennia of paleotemperature from tree-rings in the Great Basin, USA, Climate Dynamics, 42, 1517-1526, https://doi.org/10.1007/s00382-013-1911-9, 2014.

Schiff, C. J., Kaufman, D. S., Wolfe, A. P., Dodd, J., and Sharp, Z.: Late Holocene storm-trajectory changes inferred from the oxygen isotope composition of lake diatoms, south Alaska, J. Paleolimnol., 41, 189-208, https://doi.org/10.1007/s10933-0089261-z, 2009.

Schmieder, J., Fritz, S. C., Swinehart, J. B., Shinneman, A. L. C., Wolfe, A. P., Miller, G., Daniels, N., Jacobs, K. C., and Grimm, E. C.: A regional-scale climate reconstruc- 
tion of the last 4000 years from lakes in the Nebraska Sand Hills, USA, Quaternary Sci. Rev., 30, 1797-1812, https://doi.org/10.1016/j.quascirev.2011.04.011, 2011.

Shafer, D. S.: The timing of Late Quaternary monsoon precipitation maxima in the southwest United States, PhD thesis, University of Arizona, Tucson, USA, 234 pp., 1989.

Shapley, M. D., Ito, E., and Donovan, J. J.: Lateglacial and Holocene hydroclimate inferred from a groundwater flowthrough lake, Northern Rocky Mountains, USA, Holocene, 19, 523-535, https://doi.org/10.1177/0959683609104029, 2009.

Shuman, B. N. and Marsicek, J.: The structure of Holocene climate change in mid-latitude North America, Quaternary Sci. Rev., 141, 38-51, https://doi.org/10.1016/j.quascirev.2016.03.009, 2016.

Shuman, B. N., Henderson, A. K., Colman, S. M., Stone, J. R., Fritz, S. C., Stevens, L. R., Power, M. J., and Whitlock, C.: Holocene lake-level trends in the Rocky Mountains, USA, Quaternary Sci. Rev., 28, 1861-1879, https://doi.org/10.1016/j.quascirev.2009.03.003, 2009.

Shuman, B. N., Carter, G. E., Hougardy, D. D., Powers, K., and Shinker, J. J.: A north-south moisture dipole at multi-century scales in the Central and Southern Rocky Mountains, USA, during the late Holocene, Rocky Mountain Geology, 49, 33-49, https://doi.org/10.2113/gsrocky.49.1.33, 2014.

Shuman, B. N., Pribyl, P., and Buettner, J.: Hydrologic changes in Colorado during the mid-Holocene and Younger Dryas, Quaternary Res., 84, 187-199, https://doi.org/10.1016/j.yqres.2015.07.004, 2015.

Shuman, B. N., Routson, C., McKay, N., Fritz, S., Kaufman, D., Kirby, M. E., Nolan, C., Pederson, G. T., and St-Jacques, J.M.: Placing the Common Era in a Holocene context: millennial to centennial patterns and trends in the hydroclimate of North America over the past 2000 years, Clim. Past, 14, 665-686, https://doi.org/10.5194/cp-14-665-2018, 2018.

Staines-Urías, F., González-Yajimovich, O., and Beaufort, L.: Reconstruction of past climate variability and ENSO-like fluctuations in the southern Gulf of California (Alfonso Basin) since the last glacial maximum, Quaternary Res., 83, 488-501, https://doi.org/10.1016/j.yqres.2015.03.007, 2015.

Steinman, B. A., Pompeani, D. P., Abbott, M. B., Ortiz, J. D., Stansell, N. D., Finkenbinder, M. S., Mihindukulasooriya, L. N., and Hillman, A. L.: Oxygen isotope records of Holocene climate variability in the Pacific Northwest, Quaternary Sci. Rev., 142, 40-60, https://doi.org/10.1016/j.quascirev.2016.04.012, 2016.

Steponaitis, E., Andrews, A., McGee, D., Quade, J., Hsieh, Y.T., Broecker, W. S., Shuman, B. N., Burns, S. J., and Cheng, H.: Mid-Holocene drying of the U.S. Great Basin recorded in Nevada speleothems, Quaternary Sci. Rev., 127, 174-185, https://doi.org/10.1016/j.quascirev.2015.04.011, 2015.

Stone, J. R. and Fritz, S. C.: Multidecadal drought and Holocene climate instability in the Rocky Mountains, Geology, 34, 409, https://doi.org/10.1130/G22225.1, 2006.

Sundqvist, H. S., Kaufman, D. S., McKay, N. P., Balascio, N. L., Briner, J. P., Cwynar, L. C., Sejrup, H. P., Seppä, H., Subetto, D. A., Andrews, J. T., Axford, Y., Bakke, J., Birks, H. J. B., Brooks, S. J., de Vernal, A., Jennings, A. E., Ljungqvist, F. C., Rühland, K. M., Saenger, C., Smol, J. P., and Viau, A. E.: Arctic Holocene proxy climate database - new approaches to assessing geochronological accuracy and encoding climate variables,
Clim. Past, 10, 1605-1631, https://doi.org/10.5194/cp-10-16052014, 2014.

Sweeney, J., Salter-Townshend, M., Edwards, T., Buck, C. E., and Parnell, A. C.: Statistical challenges in estimating past climate changes, WIRES Comput. Stat., 10, e1437, https://doi.org/10.1002/wics.1437, 2018.

Szeicz, J. M., MacDonald, G. M., and Duk-Rodkin, A.: Late Quaternary vegetation history of the central Mackenzie Mountains, Northwest Territories, Canada, Palaeogeogr. Palaeocl., 113, 351371, https://doi.org/10.1016/0031-0182(95)00070-3, 1995.

Tingley, M. P., Craigmile, P. F., Haran, M., Li, B., Mannshardt, E., and Rajaratnam, B.: Piecing together the past: statistical insights into paleoclimatic reconstructions, Quaternary Sci. Rev., 35, 122, https://doi.org/10.1016/j.quascirev.2012.01.012, 2012.

Toney, J. L. and Anderson, R. S.: A postglacial palaeoecological record from the San Juan Mountains of Colorado USA: fire, climate and vegetation history, Holocene, 16, 505-517, https://doi.org/10.1191/0959683606hl946rp, 2006.

Upiter, L. M., Vermaire, J. C., Patterson, R. T., Crann, C. A., Galloway, J. M., Macumber, A. L., Neville, L. A., Swindles, G. T., Falck, H., Roe, H. M., and Pisaric, M. F. J.: Middle to late Holocene chironomid-inferred July temperatures for the central Northwest Territories, Canada, J. Paleolimnol., 52, 11-26, https://doi.org/10.1007/s10933-014-9775-5, 2014.

Von Storch, H., Zorita, E., Jones, J. M., Dimitriev, Y., González-Rouco, F., and Tett, S. F. B.: Reconstructing past climate from noisy data, Science, 306, 679-682, https://doi.org/10.1126/science.1096109, 2004.

Wahl, D., Byrne, R., and Anderson, L.: An 8700 year paleoclimate reconstruction from the southern Maya lowlands, Quaternary Sci. Rev., 103, 19-25, https://doi.org/10.1016/j.quascirev.2014.08.004, 2014.

White, J. M. and Mathewes, R. W.: Postglacial vegetation and climatic change in the upper Peace River district, Alberta, Can. J. Botany, 64, 2305-2318, https://doi.org/10.1139/b86-302, 1986.

Whitlock, C., Dean, W. E., Fritz, S. C., Stevens, L. R., Stone, J. R., Power, M. J., Rosenbaum, J. R., Pierce, K. L., and Bracht-Flyr, B. B.: Holocene seasonal variability inferred from multiple proxy records from Crevice Lake, Yellowstone National Park, USA, Palaeogeogr. Palaeocl., 331/332, 90-103, https://doi.org/10.1016/j.palaeo.2012.03.001, 2012.

Williams, J. W., Grimm, E. C., Blois, J. L., Charles, D. F., Davis, E. B., Goring, S. J., Graham, R. W., Smith, A. J., Anderson, M., Arroyo-Cabrales, J., Ashworth, A. C., Betancourt, J. L., Bills, B. W., Booth, R. K., Buckland, P. I., Curry, B. B., Giesecke, T., Jackson, S. T., Latorre, C., Nichols, J., Purdum, T., Roth, R. E., Stryker, M., and Takahara, H.: The Neotoma Paleoecology Database, a multiproxy, international, community-curated data resource, Quaternary Res., 89, 156177, https://doi.org/10.1017/qua.2017.105, 2018.

Winter, A., Zanchettin, D., Lachniet, M., Vieten, R., Pausata, F. S. R., Ljungqvist, F. C., Cheng, H., Edwards, R. L., Miller, T., Rubinetti, S., Rubino, A., and Taricco, C.: Initiation of a stable convective hydroclimatic regime in Central America circa 9000 years BP, Nat. Commun., 11, 716, https://doi.org/10.1038/s41467-020-14490-y, 2020.

Wong, C. I., Banner, J. L., and Musgrove, M.: Holocene climate variability in Texas, USA: An integration of existing paleoclimate data and modeling with a new, high- 
resolution speleothem record, Quaternary Sci. Rev., 127, 155173, https://doi.org/10.1016/j.quascirev.2015.06.023, 2015.

Wooller, M. J., Kurek, J., Gaglioti, B. V., Cwynar, L. C., Bigelow, N., Reuther, J. D., Gelvin-Reymiller, C., and Smol, J. P.: An 11,200 year paleolimnological perspective for emerging archaeological findings at Quartz Lake, Alaska, J. Paleolimnol., 48, 8399, https://doi.org/10.1007/s10933-012-9610-9, 2012.

Worona, M. A. and Whitlock, C.: Late Quaternary vegetation and climate history near Little Lake, central Coast Range, Oregon, Geol. Soc. Am. Bull., 107, 867-876, https://doi.org/10.1130/00167606(1995)107<0867:LQVACH>2.3.CO;2, 1995.
Yu, Z., Campbell, I. D., Campbell, C., Vitt, D. H., Bond, G. C., and Apps, M. J.: Carbon sequestration in western Canadian peat highly sensitive to Holocene wet-dry climate cycles at millennial timescales, Holocene, 13, 801-808, https://doi.org/10.1191/0959683603hl667ft, 2003. 